This document was prepared in conjunction with work accomplished under Contract No. DE-AC09-96SR18500 with the U. S. Department of Energy.

\title{
DISCLAIMER
}

This report was prepared as an account of work sponsored by an agency of the United States Government. Neither the United States Government nor any agency thereof, nor any of their employees, makes any warranty, express or implied, or assumes any legal liability or responsibility for the accuracy, completeness, or usefulness of any information, apparatus, product or process disclosed, or represents that its use would not infringe privately owned rights. Reference herein to any specific commercial product, process or service by trade name, trademark, manufacturer, or otherwise does not necessarily constitute or imply its endorsement, recommendation, or favoring by the United States Government or any agency thereof. The views and opinions of authors expressed herein do not necessarily state or reflect those of the United States Government or any agency thereof.

This report has been reproduced directly from the best available copy.

Available for sale to the public, in paper, from: U.S. Department of Commerce, National Technical Information Service, 5285 Port Royal Road, Springfield, VA 22161, phone: (800) 553-6847, fax: (703) 605-6900

email: orders@ntis.fedworld.gov

online ordering: http://www.ntis.gov/help/index.asp

Available electronically at http://www.osti.gov/bridge

Available for a processing fee to U.S. Department of Energy and its contractors, in paper, from: U.S. Department of Energy, Office of Scientific and Technical Information, P.O. Box 62, Oak Ridge, TN 37831-0062,

phone: (865)576-8401,

fax: (865)576-5728

email: $\underline{\text { reports@ adonis.osti.gov }}$ 
Key Words:

Physical Properties

Rheology

Simulant

\title{
Retention:
}

Permanent

Key WTP R\&T References:

Test Specification: Not Applicable

Test Plan: WSRC-TR-2003-00538, Rev. 0

Test Exceptions: None

R\&T Focus Area: Pulse Jet Mixers

Test Scoping Statement(s): Not Applicable

Others if appropriate: CNN 066794, Rev. 1

\section{PHYSICAL CHARACTERIZATION OF FLORIDA INTERNATIONAL UNIVERSITY SIMULANTS (U)}

\author{
Erich K. Hansen, 999-W \\ Timothy M. Jones, 999-W
}

Issue Date: APRIL 13, 2004

Westinghouse Savannah River Company

Savannah River Site

Aiken, SC 29808

Prepared for the U.S. Department of Energy Under Contract Number DE-AC09-96SR18500

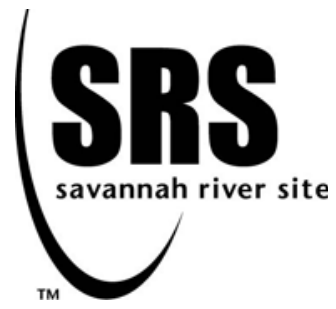


This page was intentionally left blank 
WSRC-TR-2004-00010, REV. 0

SRT-RPP-2004-00001, REV. 0

\section{TABLE OF CONTENTS}

LIST OF FIGURES .......................................................................................................................... iv

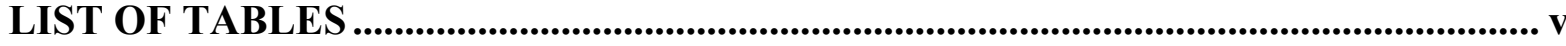

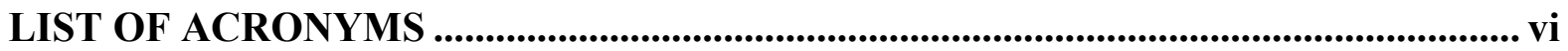

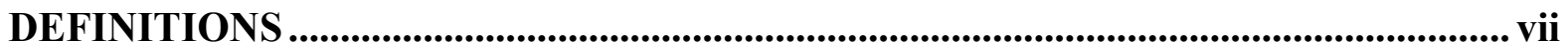

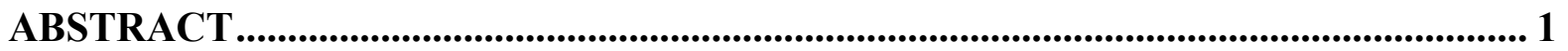

1.0 TESTING SUMMARY ............................................................................................ 3

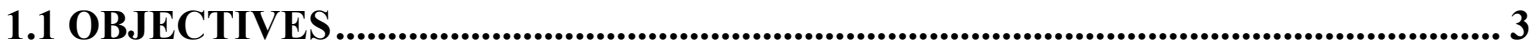

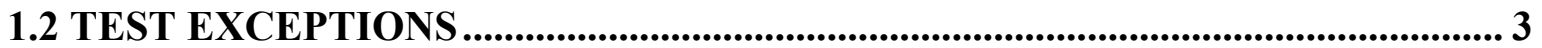

1.3 RESULTS AND PERFORMANCE AGAINST SUCCESS CRITERIA ................ 4

1.4 QUALITY REQUIREMENTS...................................................................... 10

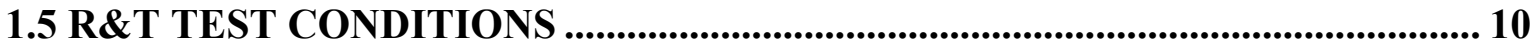

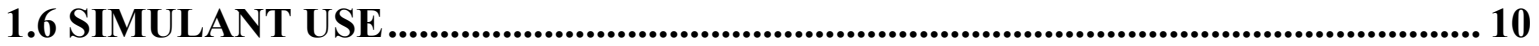

1.7 DISCREPANCIES AND FOLLOW-ON TESTS ................................................... 10

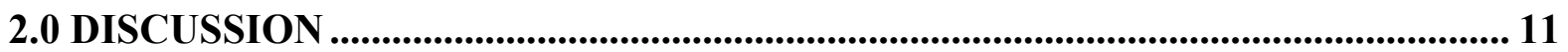

2.1 SOLIDS ANALYSIS ....................................................................................... 11

2.1.1 Density, Wt\% and Vol\% Analysis - QARD \& Clay Fluids .............................. 11

2.1.2 Density and Wt\% Total Solids - Laponite ................................................... 15

2.1.3 Settling Results of the FIU Samples........................................................................ 15

2.2 RHEOLOGY MEASUREMENT ................................................................... 16

2.2.1 Instrumentation .......................................................................................... 16

2.2.2 Flow Curve Measurements Using Concentric Geometry .................................. 17

2.2.3 Shear Strength Measurement Using Vane Geometry..................................... 19

2.2.4 Flow Curve Results ....................................................................................... 21

2.2.5 Vane Shear Strength Results.................................................................................. 22

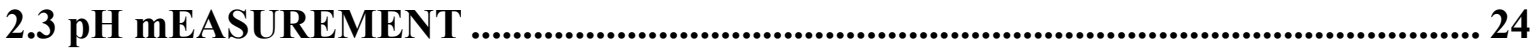

2.4 ELEMENTAL COMPOSITION ........................................................................... 25

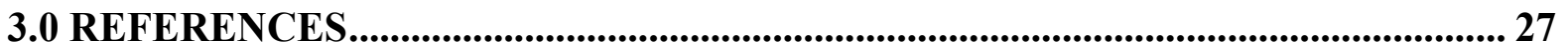

APPENDIX A - Flow Curves ............................................................................................... 29

APPENDIX B - Vane Curves....................................................................................... 37 


\section{LIST OF FIGURES}

Figure 2-1: QARD AZ-101 Samples from FIU after 3 days of Settling ................................. 12

Figure 2-2: QARD AZ-101 Samples Following Centrifuging ………………………........... 12

Figure 2-3: Drying Supernatant in Graduated Cylinders ....................................................... 14

Figure 2-4: Dried Centrifuged Solids in Cones ................................................................... 14

Figure 2-5: Normalized Settling Curves For FIU WTP-QARD-112803-Feed Sample ......... 16

Figure 2-6: Vane Geometric Requirements ……………................................................... 20

Figure 2-7: Typical Vane Torque versus Time/Displacement Curve .................................... 21

Figure 2-8: FIU WTP-QARD-112803-Feed Vane 48 hour Measurement .............................. 23

Figure A - 1: WTP-1B (Laponite) Date 11/24/03 Feed ....................................................... 30

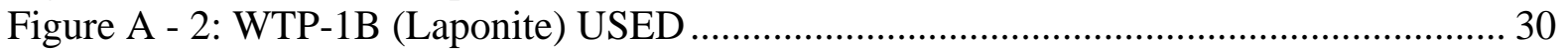

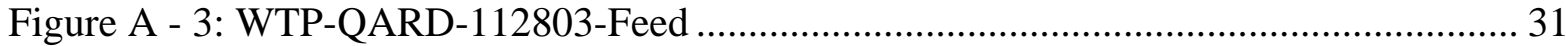

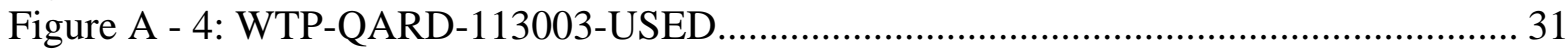

Figure A - 5: 27\% Clay Slurry From New Tote WTP Clay 1-12/6/-1 .................................. 32

Figure A - 6: 27\% Clay Slurry Used Following Ar + H2O2 Tests, WTP-Clay 1-12/6-1 ...... 32

Figure A - 7: Diluted Clay to Mimic QARD Rheology New, WTP-Clay 2-12/6-1 ................ 33

Figure A - 8: Diluted Clay After H2O2 Injection \& Release, WTP-Clay 2-12/6 ................... 33

Figure A - 9: WTP-Clay-121603-Used FIU-HCET Diluted Clay …………………............... 34

Figure A - 10: WTP-QARD-12/7-1 After $2^{\text {nd }} \mathrm{H} 2 \mathrm{O} 2$ Injection \& Release ................................ 34

Figure A - 11: WTP-QARD-12/8-2 After $3^{\text {rd }} \mathrm{H} 2 \mathrm{O} 2$ Injection \& Release..................................... 35

Figure B - 1: Shear Strength Results for FIU Laponite Samples......................................... 38

Figure B - 2: Shear Strength Results for FIU QARD Samples................................................. 38

Figure B - 3: Shear Strength Results for FIU 27\% Clay Slurry.............................................. 39

Figure B - 4: Shear Strength Results for FIU Diluted Clay to Mimic QARD Rheology ........ 39

Figure B - 5: Shear Strength Results for WTP-Clay-121603-Used FIU-HCET Diluted Clay 40

Figure B - 6: Shear Strength Results for WTP-QARD After $2^{\text {nd }}$ and $3{ }^{\text {rd }} \mathrm{H} 2 \mathrm{O} 2$ Injection and

Release Test 


\section{LIST OF TABLES}

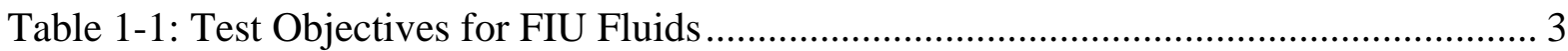

Table 1-2: FIU Physical Properties Data - Solids Analyses................................................... 5

Table 1-3: Normalized Settling Data for FIU WTP-QARD-112803-Feed Sample ................... 6

Table 1-4: FIU Physical Properties Results - Shear Strength Results....................................... 6

Table 1-5: FIU Physical Properties Results - Flow Curves Fitted to non-Newtonian

Rheological Models ..........................................................................................

Table 2-1: Fluids Sent By FIU for Physical Characterization ............................................... 11

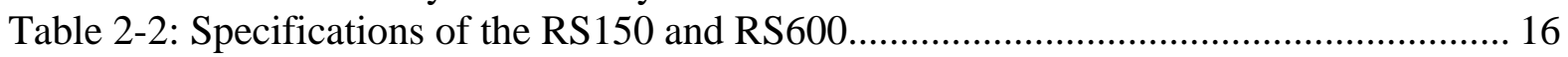

Table 2-3: Z41 Rotor Specification and Program Ramp Rates ............................................. 18

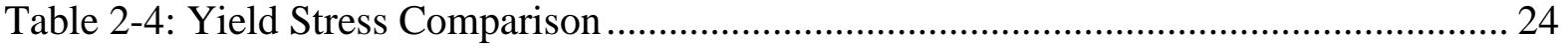

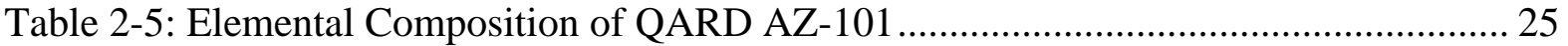




\section{LIST OF ACRONYMS}

$\begin{array}{ll}\text { DWPF } & \text { Defense Waste Processing Facility } \\ \text { FIU } & \text { Florida International University } \\ \text { H2O2 } & \text { Hydrogen Peroxide } \\ \text { ITS } & \text { Immobilization Technology Section } \\ \text { NIST } & \text { National Institute of Standards and Technology } \\ \text { N/A } & \text { Not Applicable } \\ \text { N/M } & \text { Not Measured } \\ \text { PSD } & \text { Particle Size Distribution } \\ \text { QA } & \text { Quality Assurance } \\ \text { QAPjP } & \text { Quality Assurance Project Plan } \\ \text { QARD } & \text { Quality Assurance Requirements Document } \\ \text { RPD } & \text { Relative Percent Difference } \\ \text { RPP } & \text { River Protection Project } \\ \text { R\&T } & \text { Research and Technology } \\ \text { SRS } & \text { Savannah River Site } \\ \text { SRTC } & \text { Savannah River Technology Center } \\ \text { Stdev } & \text { Standard Deviation } \\ \text { Vol\% } & \text { Volume Percent } \\ \text { Wt\% } & \text { Weight Percent } \\ \text { WTP } & \text { Waste Treatment Plant }\end{array}$


WSRC-TR-2004-00010, REV. 0

SRT-RPP-2004-00001, REV. 0

\section{DEFINITIONS}

\begin{tabular}{|c|c|c|}
\hline Variable & Definition & Units \\
\hline Apparent viscosity & The measured shear stress divided by the measured shear rate. & $\begin{array}{l}\mathrm{Pa} \cdot \mathrm{s} \\
\mathrm{cP}\end{array}$ \\
\hline Density & Mass per unit volume. & $\mathrm{g} / \mathrm{mL}$ \\
\hline Flow curve/rheogram & Plot of shear stress versus shear rate. & N/A \\
\hline Interstitial solution & Solution contained between suspended, settled, or centrifuged solids. & N/A \\
\hline Newtonian Fluid & A fluid whose apparent viscosity is independent on the shear rate. & N/A \\
\hline non-Newtonian Fluid & A fluid whose apparent viscosity is dependent on the shear rate. & N/A \\
\hline $\begin{array}{l}\text { Settled solids shear } \\
\text { strength }\end{array}$ & $\begin{array}{l}\text { The maximum shear stress as determined by the vane method for a } \\
\text { slurry sample that is allowed to settle for a specified amount of time. }\end{array}$ & $\mathrm{Pa}$ \\
\hline Solution & A liquid phase that can contain soluble solids. & grams \\
\hline Slurry & A mixture of insoluble solids and solution & grams \\
\hline Supernatant Liquid & $\begin{array}{l}\text { A liquid phase overlaying a liquid or material deposited by settling, } \\
\text { precipitation, or centrifugation. }\end{array}$ & grams \\
\hline Solids Settling Rate & $\begin{array}{l}\text { Rate at which solids in a homogenized sample settle. The change in } \\
\text { the settled solids height as a function of time. }\end{array}$ & $\mathrm{cm} / \mathrm{sec}$ \\
\hline vol $\%$ centrifuged solids & $\begin{array}{l}\text { The volume of solids layer that separates from the bulk slurry after } 1 \\
\text { hour of centrifugation at } 1000 \text { gravities divided by the total sample } \\
\text { volume on a percentage basis. These centrifuged solids will contain } \\
\text { interstitial solution. }\end{array}$ & Vol\% \\
\hline vol\% settled solids & $\begin{array}{l}\text { The percentage of the volume of slurry sample that the settled solids } \\
\text { occupy after settling for } 72 \text { hours under one gravity. These } \\
\text { centrifuged solids will contain interstitial solution. }\end{array}$ & Vol\% \\
\hline wt $\%$ centrifuge solids & $\begin{array}{l}\text { The mass of the solids layer that separates from the bulk slurry after } 1 \\
\text { hour of centrifugation at } 1000 \text { gravities divided by the total bulk } \\
\text { slurry sample mass on a percentage basis. These centrifuged solids } \\
\text { will contain interstitial solution. }\end{array}$ & wt $\%$ \\
\hline wt $\%$ oven dried solids & $\begin{array}{l}\text { The percent mass of centrifuged solids remaining after removing } \\
\text { volatiles including free water by drying at } 105+5^{\circ} \mathrm{C} \text { for } 24 \text { hours. }\end{array}$ & wt $\%$ \\
\hline $\mathrm{wt} \%$ settled solids & $\begin{array}{l}\text { The percentage (mass basis) of settled solids present in the sample. } \\
\text { Calculated by dividing the mass of the settled solids by the mass of } \\
\text { the sample. }\end{array}$ & $\mathrm{wt} \%$ \\
\hline $\begin{array}{l}\text { wt\% soluble solids in } \\
\text { supernatant }\end{array}$ & $\begin{array}{l}\text { Calculated by dividing the mass of the dried supernatant by the mass } \\
\text { of the supernatant prior to drying. }\end{array}$ & wt\% \\
\hline wt $\%$ undissolved solids & $\begin{array}{l}\text { Calculated by dividing the calculated mass of the undissolved solids } \\
\text { by the mass of the bulk solids. }\end{array}$ & wt $\%$ \\
\hline Yield Stress & $\begin{array}{l}\text { The minimum stress required to initiate flow. Determined by fitting } \\
\text { the measured flow curve using non-Newtonian rheological models } \\
\text { that have a yield stress parameter. Also determined by the vane } \\
\text { method on a homogenized sample. }\end{array}$ & $\mathrm{Pa}$ \\
\hline
\end{tabular}


WSRC-TR-2004-00010, REV. 0

SRT-RPP-2004-00001, REV. 0

This page intentionally left blank.

- viii - 


\begin{abstract}
Florida International University (FIU) shipped Laponite, clay (bentonite and kaolin blend), and Quality Assurance Requirements Document (QARD) AZ-101 simulants to the Savannah River Technology Center (SRTC) for physical characterization and to report the results. The objectives of the task were to measure the physical properties of the fluids provided by FIU and to report the results. The physical properties were measured using the approved River Protection Project (RPP) Waste Treatment Plant (WTP) characterization procedure [Ref. 1]. This task was conducted in response to the work outlined in CCN066794 [Ref. 2], authored by Gary Smith and William Graves of RPP-WTP.
\end{abstract}

The physical properties, as well as the $\mathrm{pH}$ of the FIU fluids are summarized in section 1.3. 
WSRC-TR-2004-00010, REV. 0

SRT-RPP-2004-00001, REV. 0

This page intentionally left blank. 
WSRC-TR-2004-00010, REV. 0

SRT-RPP-2004-00001, REV. 0

\subsection{TESTING SUMMARY}

\subsection{OBJECTIVES}

The objective of this task is to physically characterize the fluids provided by FIU using the characterization methods outline in the WTP characterization procedure [Ref. 1]. The fluids provided by FIU for physical analyzes included Laponite, bentonite/kaolin clay mixture (to be called "clay" from this point on), and QARD AZ-101 simulant. Laponite, when blended with water is a transparent fluid. The solids method outlined in reference 1 will be limited to bulk density and total solids analysis for Laponite fluids. Table 1-1 lists the test objectives, if the objective has been met, and what test objectives are required for a given FIU fluid.

This task was conducted in response to the objectives outlined in CCN066794 [Ref. 2], authored by Gary Smith and William Graves of RPP-WTP.

Table 1-1: Test Objectives for FIU Fluids

\begin{tabular}{|c|c|c|c|c|}
\hline \multirow{2}{*}{\multicolumn{2}{|c|}{ Test Objective (Physical Property) }} & \multirow{3}{*}{$\begin{array}{c}\begin{array}{r}\text { Objective } \\
\text { Met (Y/N) }\end{array} \\
\text { Yes }\end{array}$} & \multicolumn{2}{|c|}{ FIU FLUID } \\
\hline & & & Laponite & Clay or QARD \\
\hline \multirow{6}{*}{ 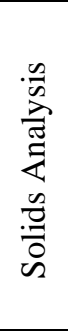 } & Bulk density & & $\mathrm{X}$ & $\mathrm{X}$ \\
\hline & Supernatant liquid density & Yes & N/A & $\mathrm{X}$ \\
\hline & $\begin{array}{c}\text { Volume percent (vol \%) } \\
\text { settled solids }\end{array}$ & Yes & N/A & $X$ \\
\hline & Settling tests & Yes & N/A & $\mathrm{X}$ \\
\hline & $\begin{array}{c}\text { Weight percent (wt \%) total } \\
\text { dried solids }\end{array}$ & Yes & $X$ & $X$ \\
\hline & wt \% undissolved solids & Yes & N/A & $\mathrm{X}$ \\
\hline \multirow{2}{*}{$\begin{array}{l}\text { dod } \\
\frac{0}{0} \\
\stackrel{0}{0} \\
\frac{0}{\alpha}\end{array}$} & $\begin{array}{c}\text { Flow curves at } 25^{\circ} \mathrm{C} \text {. Curves } \\
\text { analyzed using the Power } \\
\text { Law, Bingham Plastic and } \\
\text { Herschel-Bulkley } \\
\text { rheological models }\end{array}$ & Yes & $X$ & $X$ \\
\hline & $\begin{array}{c}\text { Vane measurements at } 25^{\circ} \mathrm{C}, \\
\text { at settled times of } \mathrm{t}=0 \\
\text { min, } 15 \mathrm{~min} ., 1 \mathrm{hr}, 2 \mathrm{hrs}, 8 \\
\text { hrs, } 16 \mathrm{hrs}, 24 \mathrm{hrs} \text { and } 48 \\
\text { hrs. }\end{array}$ & Yes & $X$ & $X$ \\
\hline
\end{tabular}

\subsection{TEST EXCEPTIONS}

There were no test exceptions issued to this task. 
WSRC-TR-2004-00010, REV. 0

SRT-RPP-2004-00001, REV. 0

\subsection{RESULTS AND PERFORMANCE AGAINST SUCCESS CRITERIA}

No success criteria were specified in reference [2]. The requirement of this task is to provide physical characterization data of the FIU samples shipped to SRTC for analysis.

The solids (wt $\%$, vol\%, and density) analyses and $\mathrm{pH}$ results of the FIU samples are provided in Table 1-2. The only fluid to show any settling within 3 days was the WTP-QARD112803-Feed sample and it is shown in Table 1-3. Details of the solids analyses are provided in section 2.1 and $\mathrm{pH}$ in section 2.3.

The vane and flow curve analyses are shown in Table 1-4 and Table 1-5 respectively. A comparison of the initial vane measurement $(\mathrm{t}=0)$ and Bingham Plastic yield stress are comparable (Table 2-4). Details of the rheological measurements and analyses are provided in section 2.2.

Section 2.4 provides the chemical composition of the QARD AZ-101 simulant. There was no criterion in providing this information. 
WSRC-TR-2004-00010, REV. 0

SRT-RPP-2004-00001, REV. 0

Table 1-2: FIU Physical Properties Data - Solids Analyses

\begin{tabular}{|c|c|c|c|c|c|c|c|c|c|c|c|c|}
\hline Physical Property & Units & 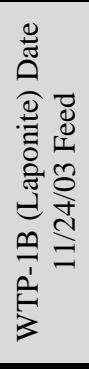 & 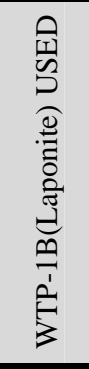 & 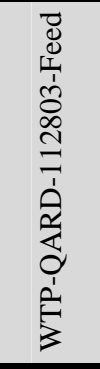 & 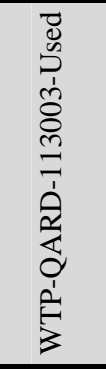 & 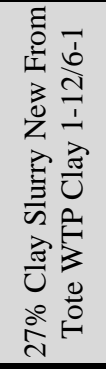 & 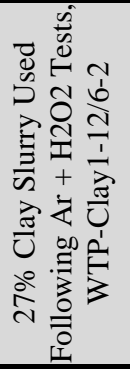 & 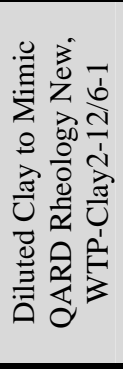 & 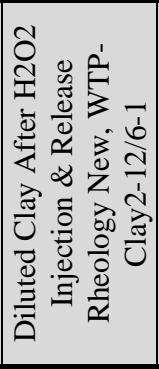 & 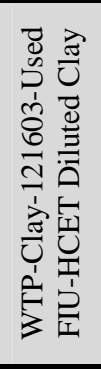 & 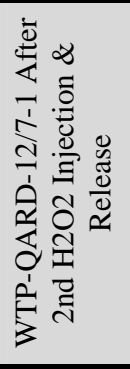 & 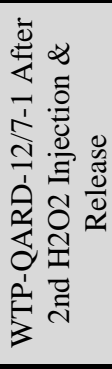 \\
\hline $\begin{array}{l}\text { Sodium concentration of LAW } \\
\text { waste or pretreated waste }\end{array}$ & Molar & N/A & N/A & N/A & N/A & N/A & N/A & N/A & N/A & N/A & N/A & N/A \\
\hline $\mathrm{pH}$ & $\mathrm{pH}$ & 9.01 & 8.98 & 12.71 & 12.71 & 6.72 & 6.92 & 6.52 & 6.79 & 2.81 & 12.75 & 12.77 \\
\hline Solid phases present & yes/no & yes & yes & yes & yes & yes & yes & yes & yes & yes & yes & yes \\
\hline Density - Bulk slurry & $\mathrm{g} / \mathrm{mL}$ & 0.99 & 0.98 & 1.20 & 1.19 & 1.19 & 1.19 & 1.17 & 1.17 & 1.17 & 1.21 & 1.18 \\
\hline Density - settled solids & $\mathrm{g} / \mathrm{mL}$ & $\mathrm{N} / \mathrm{M}$ & $\mathrm{N} / \mathrm{M}$ & 1.30 & 1.19 & 1.19 & 1.19 & 1.17 & 1.17 & 1.17 & 1.18 & 1.18 \\
\hline Density - centrifuged solids & $\mathrm{g} / \mathrm{mL}$ & $\mathrm{N} / \mathrm{M}$ & $\mathrm{N} / \mathrm{M}$ & 1.28 & 1.31 & 1.02 & 1.14 & 1.16 & 1.17 & 1.36 & 1.33 & 1.27 \\
\hline Density - supernatant liquid & $\mathrm{g} / \mathrm{mL}$ & $\mathrm{N} / \mathrm{M}$ & $\mathrm{N} / \mathrm{M}$ & 1.00 & 1.03 & 0.93 & 0.95 & 0.92 & 0.95 & 0.99 & 1.02 & 0.99 \\
\hline Vol. \% settled solids after 72 hours & $\%$ & 100 & 100 & 85.82 & 100 & 100 & 100 & 100 & 100 & 100 & 100 & 100.00 \\
\hline Vol. $\%$ centrifuged solids & $\%$ & $\mathrm{~N} / \mathrm{M}$ & $\mathrm{N} / \mathrm{M}$ & 70.93 & 61.57 & 96.06 & 98.87 & 92.82 & 93.92 & 46.98 & 60.42 & 60.76 \\
\hline Wt $\%$ total dried solids & $\%$ & 1.42 & 1.38 & 20.78 & 20.31 & 25.26 & 25.69 & 22.82 & 22.58 & 21.75 & 20.09 & 19.91 \\
\hline Wt $\%$ centrifuged solids & $\%$ & $\mathrm{~N} / \mathrm{M}$ & $\mathrm{N} / \mathrm{M}$ & 76.00 & 67.81 & 81.67 & 94.86 & 92.33 & 93.68 & 54.81 & 66.36 & 65.53 \\
\hline Wt $\%$ oven dried solids & $\%$ & $\mathrm{~N} / \mathrm{M}$ & $\mathrm{N} / \mathrm{M}$ & 26.49 & 28.72 & 30.84 & 26.68 & 24.72 & 23.91 & 39.51 & 29.03 & 29.08 \\
\hline Wt \% undissolved solids & $\%$ & $\mathrm{~N} / \mathrm{M}$ & $\mathrm{N} / \mathrm{M}$ & 18.60 & 18.17 & 24.93 & 23.45 & 22.71 & 21.67 & 21.59 & 18.06 & 17.87 \\
\hline Wt \% dissolved solids & $\%$ & $\mathrm{~N} / \mathrm{M}$ & $\mathrm{N} / \mathrm{M}$ & 2.18 & 2.14 & 0.34 & 2.24 & 0.11 & 0.92 & 0.16 & 2.03 & 2.05 \\
\hline
\end{tabular}


WSRC-TR-2004-00010, REV. 0

SRT-RPP-2004-00001, REV. 0

Table 1-3: Normalized Settling Data for FIU WTP-QARD-112803-Feed Sample

\begin{tabular}{|c|c|}
\hline Measurement Interval & $\begin{array}{c}\text { Averaged Normalized } \\
\text { Interface Level }\end{array}$ \\
\hline $0,5,10,15,20,20,40,50$ minutes & 1.0 \\
\hline 1 and 2 hours & 1.0 \\
\hline 3 hours & 0.985 \\
\hline 4 hours & 0.974 \\
\hline 5 hours & 0.967 \\
\hline 6 hours & 0.959 \\
\hline 24 hours & 0.929 \\
\hline 48 hours & 0.929 \\
\hline 72 hours final settled $\left(\mathrm{V}_{\mathrm{SS}}\right)$ & 0.922 \\
\hline
\end{tabular}

Table 1-4: FIU Physical Properties Results - Shear Strength Results

\begin{tabular}{|c|c|c|c|c|c|c|c|c|c|c|c|}
\hline \multirow[b]{2}{*}{$\begin{array}{c}\text { Time of } \\
\text { Result after } \\
\text { sample has } \\
\text { been } \\
\text { homogenized }\end{array}$} & \multicolumn{11}{|c|}{ Shear Strength (Pa) } \\
\hline & 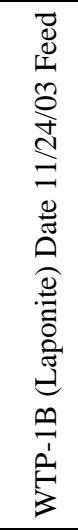 & 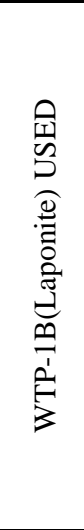 & 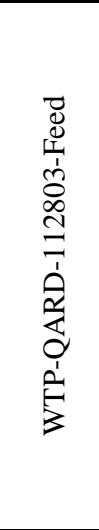 & 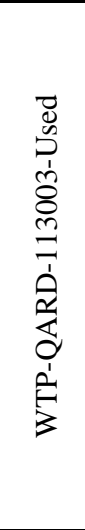 & 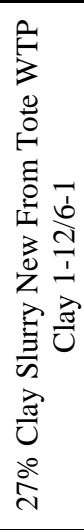 & 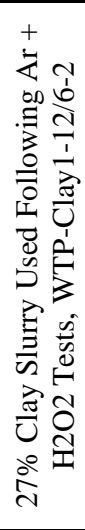 & 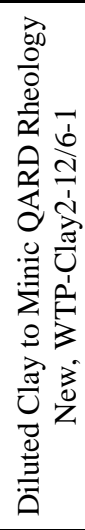 & 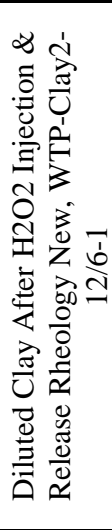 & 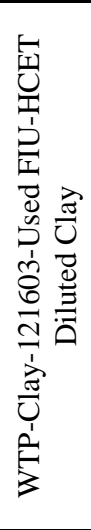 & 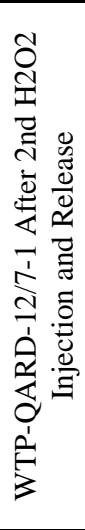 & 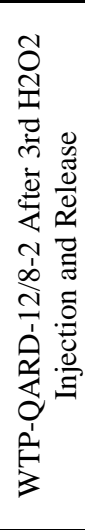 \\
\hline 0 minutes & 16.3 & 16.0 & 3.4 & 18.6 & 19.7 & 13.7 & 8.3 & 6.1 & 17.0 & 14.6 & 13.7 \\
\hline 15 minute & 32.1 & 23.6 & 3.5 & 19.3 & 22.6 & 20.3 & 10.3 & 8.9 & 17.0 & 15.9 & 14.6 \\
\hline 1 hour & 41.5 & 36.1 & 3.7 & 21.5 & 25.7 & 23.5 & 12.0 & 10.1 & 17.5 & 19.0 & 17.4 \\
\hline 2 hours & 46.6 & 42.5 & 4.2 & 22.2 & 25.1 & 22.3 & 11.0 & 8.9 & 17.2 & 21.5 & 18.3 \\
\hline 4 hours & 52.1 & 48.8 & 6.0 & 23.7 & 28.3 & 25.8 & 12.9 & 10.4 & 18.4 & 23.5 & 20.6 \\
\hline 8 hours & 53.1 & 50.9 & 19.1 & 31.2 & 25.9 & 26.0 & 11.0 & 11.2 & 20.1 & 21.8 & 18.9 \\
\hline 16 hours & 64.6 & 58.4 & 32.0 & 31.9 & 31.2 & 33.7 & 11.2 & 11.5 & 19.5 & 26.8 & 24.1 \\
\hline 24 hours & 69.6 & 66.0 & 54.4 & 41.2 & 32.1 & 29.7 & 14.6 & 13.2 & 23.6 & 22.2 & 22.7 \\
\hline 48 hours & 75.1 & 70.9 & 151.3 & 38.2 & 39.1 & 35.5 & 17.5 & 16.5 & 27.9 & 27.9 & 28.1 \\
\hline
\end{tabular}


WSRC-TR-2004-00010, REV. 0

SRT-RPP-2004-00001, REV. 0

Table 1-5: FIU Physical Properties Results - Flow Curves Fitted to non-Newtonian Rheological Models

\begin{tabular}{|c|c|c|c|c|c|c|c|c|c|c|c|c|c|c|c|}
\hline & \multicolumn{3}{|c|}{$\begin{array}{l}\text { WTP-1B (Laponite) Date } \\
\text { 11/24/03 Feed }\end{array}$} & \multicolumn{3}{|c|}{$\begin{array}{l}\text { WTP-1B (Laponite) Date } \\
\text { 11/24/03 Used }\end{array}$} & \multicolumn{3}{|c|}{ WTP-QARD-112803-Feed } & \multicolumn{3}{|c|}{ WTP-QARD-113003-Used } & \multicolumn{3}{|c|}{$\begin{array}{l}27 \% \text { Clay Slurry New From } \\
\text { Tote WTP-Clay1-12/6-1 }\end{array}$} \\
\hline & $1^{\mathrm{st}}$ & $2^{\text {nd }}$ & Avg & $1^{\mathrm{st}}$ & $2^{\text {nd }}$ & Avg & $1^{\mathrm{st}}$ & $2^{\text {nd }}$ & Avg & $1^{\mathrm{st}}$ & $2^{\text {nd }}$ & Avg & $1^{\mathrm{st}}$ & $2^{\text {nd }}$ & Avg \\
\hline \multicolumn{16}{|l|}{ Ostwald (or Power Law): } \\
\hline $\mathrm{m}-$ consistency coefficient $\left(\mathrm{Pa}-\mathrm{sec}^{\mathrm{n}}\right)$ & 2.324 & 2.293 & 2.309 & 2.142 & 2.253 & 2.198 & 0.2366 & 0.2454 & 0.241 & 8.170 & 8.124 & 8.147 & 3.860 & 3.828 & 3.844 \\
\hline $\mathrm{n}$ - power law exponent & 0.273 & 0.276 & 0.275 & 0.290 & 0.281 & 0.285 & 0.632 & 0.619 & 0.625 & 0.163 & 0.163 & 0.163 & 0.330 & 0.332 & 0.331 \\
\hline $\mathrm{R}^{2}-$ correlation coefficient & 0.9580 & 0.9582 & & 0.9588 & 0.9563 & & 0.9906 & 0.9904 & & 0.9426 & 0.9446 & & 0.9579 & 0.9612 & \\
\hline \multicolumn{16}{|l|}{ Bingham Plastic: } \\
\hline$\tau_{\mathrm{BP}}-$ Bingham yield stress $(\mathrm{Pa})$ & 7.63 & 7.66 & 7.64 & 7.59 & 7.68 & 7.63 & 3.41 & 3.38 & 3.39 & 16.45 & 16.31 & 16.38 & 16.23 & 16.25 & 16.24 \\
\hline$\eta_{\mathrm{BP}}-$ Bingham Plastic viscosity (cP) & 8.82 & 8.95 & 8.88 & 9.43 & 9.19 & 9.31 & 16.12 & 15.12 & 15.62 & 10.51 & 10.46 & 10.49 & 24.36 & 24.59 & 24.48 \\
\hline $\mathrm{R}^{2}$ - correlation coefficient & 0.9580 & 0.9582 & & 0.9588 & 0.9563 & & 0.9962 & 0.9962 & & 0.9514 & 0.9465 & & 0.9908 & 0.9888 & \\
\hline \multicolumn{16}{|l|}{ Herschel-Bulkley: } \\
\hline$\tau_{\mathrm{HB}}-$ Herschel-Bulkley yield stress $(\mathrm{Pa})$ & 4.50 & 4.47 & 4.48 & 4.26 & 4.28 & 4.27 & 2.42 & 2.40 & 2.41 & 13.38 & 12.91 & 13.15 & 13.42 & 13.07 & 13.25 \\
\hline $\begin{array}{l}\text { k - Herschel-Bulkely consistency coefficient (Pa- } \\
\left.\qquad \sec ^{b}\right)\end{array}$ & 0.398 & 0.405 & 0.402 & 0.418 & 0.448 & 0.433 & 0.049 & 0.048 & 0.048 & 0.329 & 0.404 & 0.366 & 0.155 & 0.184 & 0.170 \\
\hline b - Hershel-Bulkely power law exponent & 0.483 & 0.483 & 0.483 & 0.485 & 0.473 & 0.479 & 0.845 & 0.838 & 0.841 & 0.530 & 0.502 & 0.516 & 0.743 & 0.720 & 0.731 \\
\hline $\mathrm{R}^{2}-$ correlation coefficient & 0.9992 & 0.9990 & & 0.9990 & 0.9992 & & 0.9990 & 0.9994 & & 0.9805 & 0.9787 & & 0.9996 & 0.9994 & \\
\hline
\end{tabular}


WSRC-TR-2004-00010, REV. 0

SRT-RPP-2004-00001, REV. 0

Table 1-5: FIU Physical Properties Results - Flow Curves Fitted to non-Newtonian Rheological Models (Continued)

\begin{tabular}{|c|c|c|c|c|c|c|c|c|c|c|c|c|}
\hline & \multicolumn{3}{|c|}{$\begin{array}{c}\text { 27\% Clay Slurry Used } \\
\text { Following Ar }+\mathrm{H} 2 \mathrm{O} 2 \\
\text { Tests, WTP-Clay1-12/6- } \\
2\end{array}$} & \multicolumn{3}{|c|}{$\begin{array}{c}\text { Diluted Clay To Mimic } \\
\text { QARD Rheology New, } \\
\text { WTP-Clay2-12/6-1 }\end{array}$} & \multicolumn{3}{|c|}{$\begin{array}{c}\text { Diluted Clay After } \\
\text { H2O2 Injection \& } \\
\text { Release Rheology New, } \\
\text { WTP-Clay2-12/6-1 }\end{array}$} & \multicolumn{3}{|c|}{$\begin{array}{l}\text { WTP-Clay-121603-Used1 } \\
\text { FIU-HCET Diluted Clay }\end{array}$} \\
\hline & $1^{\mathrm{st}}$ & $2^{\text {nd }}$ & Avg & $1^{\mathrm{st}}$ & $2^{\text {nd }}$ & Avg & $1^{\mathrm{st}}$ & $2^{\text {nd }}$ & Avg & $1^{\mathrm{st}}$ & $2^{\text {nd }}$ & Avg \\
\hline \multicolumn{13}{|l|}{ Ostwald (or Power Law): } \\
\hline $\mathrm{m}-$ the consistency coefficient $\left(\mathrm{Pa}-\mathrm{sec}^{\mathrm{n}}\right)$ & 1.778 & 1.792 & 1.785 & 1.113 & 0.953 & 1.033 & 0.539 & 0.538 & 0.538 & 17.887 & 17.760 & 17.824 \\
\hline $\mathrm{n}$ - the power law exponent & 0.439 & 0.440 & 0.439 & 0.433 & 0.456 & 0.444 & 0.530 & 0.529 & 0.530 & 0.135 & 0.138 & 0.137 \\
\hline $\mathrm{R}^{2}-$ correlation coefficient & 0.9777 & 0.9781 & & 0.9841 & 0.9851 & & 0.9904 & 0.9906 & & 0.9900 & 0.9890 & \\
\hline \multicolumn{13}{|l|}{ Bingham Plastic: } \\
\hline$\tau_{\mathrm{BP}}$ - the Bingham yield stress $(\mathrm{Pa})$ & 11.97 & 12.11 & 12.04 & 7.29 & 6.89 & 7.09 & 5.24 & 5.20 & 5.22 & 31.00 & 31.21 & 31.11 \\
\hline$\eta_{\mathrm{BP}}$ - the Bingham Plastic viscosity $(\mathrm{cP})$ & 27.55 & 27.84 & 27.70 & 16.42 & 16.84 & 16.63 & 17.02 & 16.90 & 16.96 & 19.95 & 20.53 & 20.24 \\
\hline $\mathrm{R}^{2}$ - correlation coefficient & 0.9082 & 0.9910 & & 0.9855 & 0.9874 & & 0.9900 & 0.9886 & & 0.8892 & 0.8768 & \\
\hline \multicolumn{13}{|l|}{ Herschel-Bulkley: } \\
\hline$\tau_{\mathrm{HB}}-$ the Herschel-Bulkley yield stress $(\mathrm{Pa})$ & 8.84 & 8.96 & 8.90 & 4.74 & 4.51 & 4.62 & 3.18 & 3.05 & 3.11 & 13.64 & 9.23 & 11.43 \\
\hline $\begin{array}{l}\mathrm{k} \text { - the Herschel-Bulkely consistency } \\
\text { coefficient }\left(\mathrm{Pa}-\mathrm{sec}^{\mathrm{b}}\right)\end{array}$ & 0.173 & 0.172 & 0.173 & 0.163 & 0.144 & 0.154 & 0.117 & 0.125 & 0.121 & 7.009 & 10.220 & 8.615 \\
\hline b - the Hershel-Bulkely power law exponent & 0.744 & 0.746 & 0.745 & 0.681 & 0.702 & 0.692 & 0.732 & 0.722 & 0.727 & 0.221 & 0.187 & 0.204 \\
\hline $\mathrm{R}^{2}-$ correlation coefficient & 0.9992 & 0.9992 & & 0.9996 & 0.9994 & & 0.9992 & 0.9990 & & 0.9930 & 0.9898 & \\
\hline
\end{tabular}


WSRC-TR-2004-00010, REV. 0

SRT-RPP-2004-00001, REV. 0

Table 1-5: FIU Physical Properties Results - Flow Curves Fitted to non-Newtonian Rheological Models (Continued)

\begin{tabular}{|c|c|c|c|c|c|c|}
\hline & \multirow{2}{*}{\multicolumn{3}{|c|}{$\begin{array}{l}\text { WTP-QARD-12/7-1 } \\
\text { After 2nd H2O2 } \\
\text { Injection \& Release }\end{array}$}} & \multirow{2}{*}{\multicolumn{3}{|c|}{$\begin{array}{l}\text { WTP-QARD-12/8-2 } \\
\text { After 3rd H2O2 } \\
\text { Injection \& Release }\end{array}$}} \\
\hline & & & & & & \\
\hline & $1^{\text {st }}$ & $2^{\text {nd }}$ & Avg & $1^{\mathrm{st}}$ & $2^{\text {nd }}$ & Avg \\
\hline \multicolumn{7}{|l|}{ Ostwald (or Power Law): } \\
\hline $\mathrm{m}-$ the consistency coefficient $\left(\mathrm{Pa}-\mathrm{sec}^{\mathrm{n}}\right)$ & 6.71 & 6.56 & 6.635 & 6.278 & 6.364 & 6.321 \\
\hline $\mathrm{n}$ - the power law exponent & 0.1726 & 0.1764 & 0.175 & 0.1783 & 0.1774 & 0.178 \\
\hline $\mathrm{R}^{2}-$ correlation coefficient & 0.9698 & 0.9725 & & 0.971 & 0.9723 & \\
\hline \multicolumn{7}{|l|}{ Bingham Plastic: } \\
\hline$\tau_{\mathrm{BP}}-$ the Bingham yield stress $(\mathrm{Pa})$ & 14.03 & 13.98 & 14.01 & 13.5 & 13.55 & 13.53 \\
\hline$\eta_{\mathrm{BP}}$ - the Bingham Plastic viscosity (cP) & 9.651 & 9.752 & 9.70 & 9.531 & 9.702 & 9.62 \\
\hline $\mathrm{R}^{2}-$ correlation coefficient & 0.9801 & 0.9744 & & 0.9813 & 0.9694 & \\
\hline \multicolumn{7}{|l|}{ Herschel-Bulkley: } \\
\hline$\tau_{\mathrm{HB}}-$ the Herschel-Bulkley yield stress $(\mathrm{Pa})$ & 11.61 & 11.05 & 11.33 & 11.19 & 10.14 & 10.67 \\
\hline $\begin{array}{l}\mathrm{k} \text { - the Herschel-Bulkely consistency } \\
\text { coefficient }\left(\mathrm{Pa}-\mathrm{sec}^{\mathrm{b}}\right)\end{array}$ & 0.2262 & 0.3218 & 0.274 & 0.2072 & 0.447 & 0.327 \\
\hline b - the Hershel-Bulkely power law exponent & 0.567 & 0.5225 & 0.545 & 0.5771 & 0.4791 & $\mathbf{0 . 5 2 8}$ \\
\hline $\mathrm{R}^{2}$ - correlation coefficient & 0.9926 & 0.9893 & & 0.9932 & 0.9867 & \\
\hline
\end{tabular}




\subsection{QUALITY REQUIREMENTS}

This work was conducted in accordance with the RPP-WTP QA requirements specified for work conducted by SRTC as identified in DOE IWO MOSRLE60. SRTC has provided matrices to WTP demonstrating compliance of the SRTC QA program with the requirements specified by WTP [Ref. 3]. Specific information regarding the compliance of the SRTC QA program with RW-0333P, Revision 10, NQA-1 1989, Part 1, Basic and Supplementary Requirements and NQA-2a 1990, Subpart 2.7 is contained in these matrices.

The Task Technical and Quality Assurance Plan used to conduct this work are specified in WSRC-TR-2003-00538, Rev. 0.

\subsection{R\&T TEST CONDITIONS}

No WTP R\&T test conditions were established for the characterization tests performed under this task. Work was performed, following the methodology provided in reference 1.

\subsection{SIMULANT USE}

The samples characterized in this task were provided by FIU and authorized by WTP for physical characterization. Simulant use by FIU was determined by WTP as appropriate.

\subsection{DISCREPANCIES AND FOLLOW-ON TESTS}

No discrepancies were encountered in the performance of this task. No follow-on testing is necessary. 


\subsection{DISCUSSION}

SRTC was tasked to measure the physical properties of the fluids being tested by FIU to support WTP programs at FIU. The physical properties will be characterized by the methods as described in reference 1. Listed in Table 2-1 is the chronological order in which the fluids were sent by FIU for physical characterization.

Table 2-1: Fluids Sent By FIU for Physical Characterization

\begin{tabular}{|c|c|l|}
\hline$\#$ & Fluid & Sample Identification \\
\hline 1 & Laponite & WTP-1B (Laponite) Date 11/24/03 Feed \\
\hline 2 & Laponite & WTP-1B (Laponite) USED \\
\hline 3 & QARD & WTP-QARD-112803-Feed \\
\hline 4 & QARD & WTP-QARD-113003-Used \\
\hline 5 & Clay & 27\% Clay Slurry New From Tote WTP Clay 1-12/6-1 \\
\hline 6 & Clay & 27\% Clay Slurry Used Following AR + H2O2 Test, WTP-Clay 1-12/6-2 \\
\hline 7 & Clay & Diluted Clay to Mimic QARD Rheology New, WTP-Clay 2-12/6-1 \\
\hline 8 & Clay & Diluted Clay After H2O2 Injection \& Release Rheology, WTP-Clay2-12/6-1 \\
\hline 9 & Clay & WTP-Clay-121603-Used FIU-HCET Diluted Clay \\
\hline 10 & QARD & WTP-QARD-12/7-1 After 2 ${ }^{\text {nd }}$ H2O2 Injection and Release \\
\hline 11 & QARD & WTP-QARD-12/8-2 After $3^{\text {rd }}$ H2O2 Injection and Release \\
\hline
\end{tabular}

The physical properties that were measured for the fluids provided by FIU are listed in Table 1-1. Section 2.1 discusses the methods, results and observation made when performing the solids analysis. Section 2.2 discusses the methods, results and observations made when performing the rheological analysis. Section 2.3 discusses how the $\mathrm{pH}$ of the fluids was measured.

Section 2.4 discusses the chemical composition of QARD AZ-101 fluid. Reference 2 does not state that the chemical compositions of the fluids needed to be analyzed.

\subsection{SOLIDS ANALYSIS}

\subsubsection{Density, Wt\% and Vol\% Analysis - QARD \& Clay Fluids}

Between 8 to $10 \mathrm{~mL}$ of the FIU fluid (denoted as slurry) was placed into a $10 \mathrm{~mL}$ volumetric graduated centrifuge cone. The mass $\left(\mathrm{M}_{\mathrm{B}}\right)$ and volume of the slurry were recorded. The sample was then allowed to settle for at least 3 days. A detailed description of the settling test is provided in section 2.1.3. After the settling test was completed, the total volume $\left(\mathrm{V}_{\mathrm{SB}}\right)$ and volume of settled solids $\left(\mathrm{V}_{\mathrm{SS}}\right)$ were recorded. The error associated with this measurement is the total volume, which may be under or over estimated, based on how the meniscus was read (Figure 2-1). Additionally, material stuck to the sides of the tube would decrease the level of settled slurry and the total volume (Figure 2-1). This was evident for all the slurries characterized. Finally, if the material adheres to the sides of the tube such that no reading can be obtained, as is the case for the WTP-QARD-113003-Used sample (Figure 2-1), the results are not useful. 


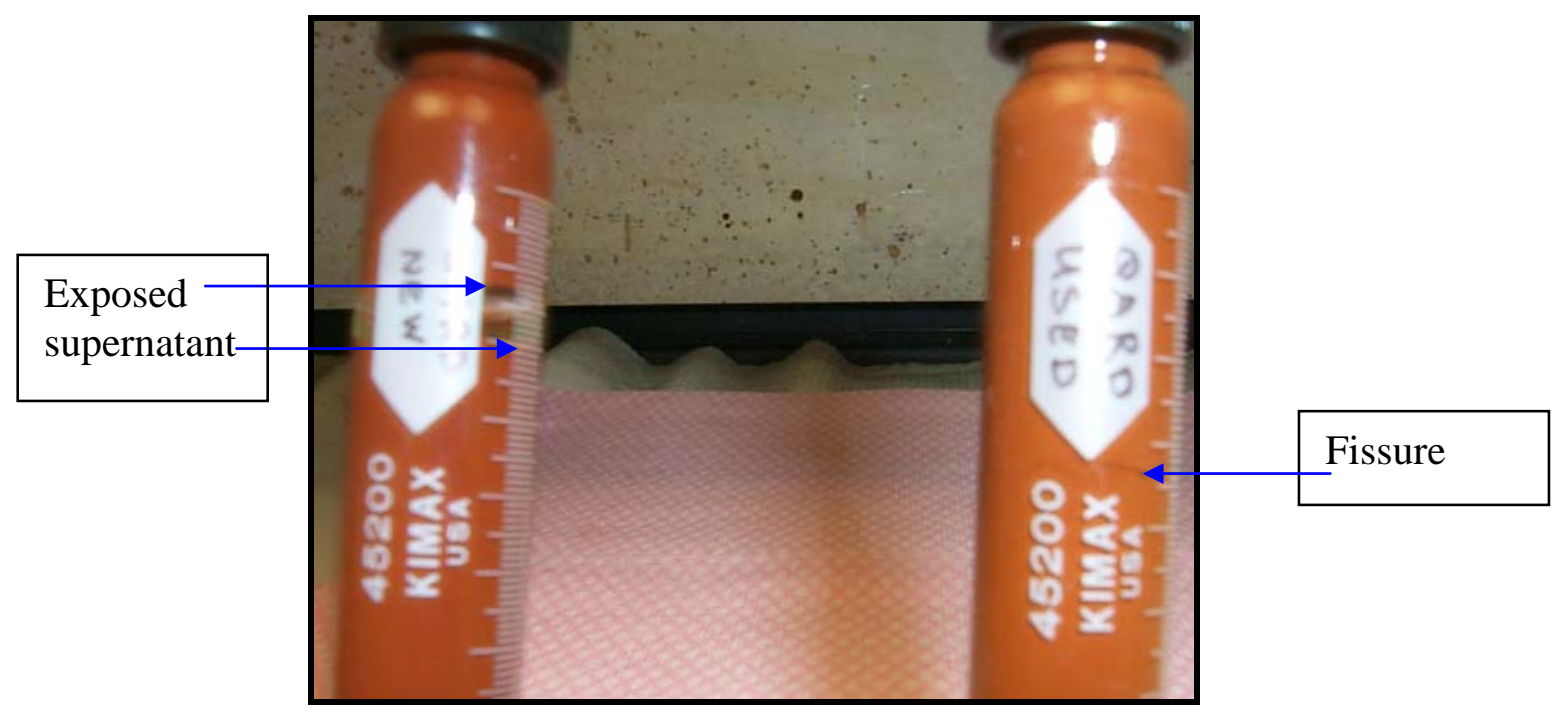

Figure 2-1: QARD AZ-101 Samples from FIU after 3 days of Settling

The settled slurry was then centrifuged at approximately one thousand times the force of gravity for 60 minutes. An additional 60 minutes of centrifugation was performed on some of the clay samples, if adequate separation did not occur during the initial centrifugation. The total volume after centrifuging $\left(\mathrm{V}_{\mathrm{B}}\right)$ and solids volume after centrifuging $\left(\mathrm{V}_{\mathrm{CS}}\right)$ were recorded. The bulk density $\left(\rho_{B}=M_{B} / V_{B}\right)$, vol\% settled solids $\left(P_{\mathrm{VSS}}=V_{S S} / V_{B} \times 100 \%\right)$ and vol\% centrifuged solids $\left(\mathrm{P}_{\mathrm{VCS}}=\mathrm{V}_{\mathrm{CS}} / \mathrm{V}_{\mathrm{B}} \times 100 \%\right)$ were then calculated. The error associated with this measurement is the total volume, which may be under or over estimated, based on how the meniscus was read (Figure 2-2). Additionally, material stuck to the sides of the tube would decrease the volume of settled slurry and the total volume (Figure 2-2), thus overestimating the density.

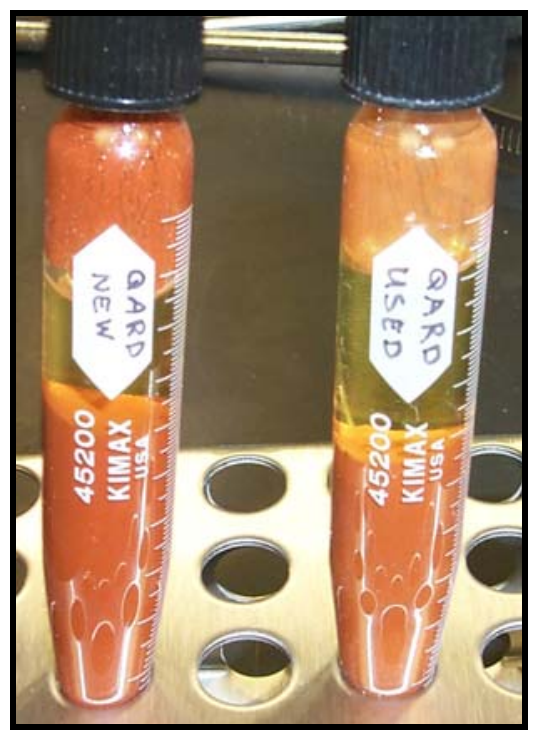

Figure 2-2: QARD AZ-101 Samples Following Centrifuging 
The transparent supernatant was then transferred from the centrifuge cone to a graduated cylinder. The mass $\left(\mathrm{M}_{\mathrm{S}}=\mathrm{M}_{\mathrm{VL}}\right.$ in this case $)$ and volume $\left(\mathrm{V}_{\mathrm{S}}\right)$ of the supernatant and the mass $\left(M_{C S}\right)$ of centrifuged solids were recorded. The density of the supernatant $\left(\rho_{S}=M_{S} / V_{S}\right)$, the density of centrifuged solids $\left(\rho_{\mathrm{CS}}=\mathrm{M}_{\mathrm{CS}} / \mathrm{V}_{\mathrm{CS}}\right)$, the wt $\%$ centrifuged solids $\left(\mathrm{P}_{\mathrm{MCS}}=\mathrm{M}_{\mathrm{CS}} / \mathrm{M}_{\mathrm{B}} \mathrm{X}\right.$ $100 \%)$, the mass of settled solids $\left(\mathrm{M}_{\mathrm{SS}}=\mathrm{M}_{\mathrm{B}}-\rho_{\mathrm{S}} \mathrm{x}\left(\mathrm{V}_{\mathrm{SB}}-\mathrm{V}_{\mathrm{SS}}\right)\right.$ ), density of settled solids $\left(\rho_{\mathrm{SS}}\right.$ $\left.=\mathrm{M}_{\mathrm{SS}} / \mathrm{V}_{\mathrm{SS}}\right)$ and $\mathrm{wt} \%$ of settled solids $\left(\mathrm{P}_{\mathrm{SS}}=\mathrm{M}_{\mathrm{SS}} / \mathrm{M}_{\mathrm{B}} \times 100 \%\right)$ were then calculated. Variables that could potentially impact these results are:

- Air entrainment. Air could potentially be released during centrifuging. Differences in the final volume between the settling and centrifuged total volume could indicate such a condition exists. It is also possible the bubbles would not be released by centrifuging. Correction to the data set requires making assumptions that could bias the results.

- The volume of supernatant transferred from the cone to the graduated cylinder is not $100 \%$ (i.e., not all the free standing supernatant was transferred). This would bias the wt $\%$ of undissolved solids high. The opposite would be true if insoluble solids were transferred for supernatant analysis.

- Volume of supernatant, as read on the graduated cylinder, could bias the density result high or low. This would impact the settled solids results.

The graduated cylinder containing the supernatant and the centrifuged cone containing the centrifuged solids were then placed into a drying oven at $90^{\circ} \mathrm{C}$. Samples remained in the oven overnight (Figure 2-3 and Figure 2-4). The oven temperature was then increased to $105^{\circ} \mathrm{C}$ and the sample was maintained in the oven until the dried weight stabilized (took approximately 2 days). The mass of the dried supernatant solids $\left(\mathrm{M}_{\mathrm{DCL}}\right)$ and mass of the dried centrifuged solids $\left(\mathrm{M}_{\mathrm{DCS}}\right)$ were recorded. Assuming that the mass lost is only water, the wt\% soluble solids in the supernatant $\left(\mathrm{P}_{\mathrm{SSS}}=\mathrm{M}_{\mathrm{DCL}} / \mathrm{M}_{\mathrm{VL}} \times 100 \%\right)$, wt\% total dried solids (equation 3.1), wt. \% oven dried solids $\left(\mathrm{P}_{\mathrm{ODS}}=\mathrm{M}_{\mathrm{DCS}} / \mathrm{M}_{\mathrm{CS}} \mathrm{x} 100 \%\right)$ and wt $\%$ UDS (equation 3.2) were then calculated.

$$
\begin{aligned}
& P_{M T S}=\left[\frac{P S S S}{100 \%} \cdot \frac{M_{S}}{M_{B}}+\frac{M_{D C S}}{M_{B}}\right] \times 100 \% \\
& P_{M U S}=\left[1-\frac{1-\frac{M_{D C L}}{M_{C S}}}{1-\frac{M_{D C L}}{M_{V L}}}\right] \times \frac{M_{C S}}{M_{B}} \times 100 \%
\end{aligned}
$$




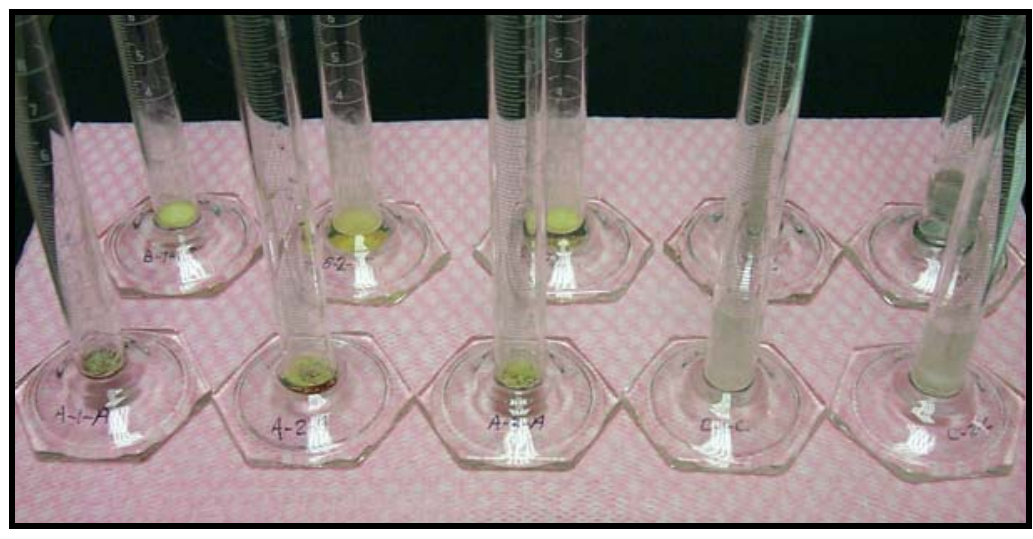

Figure 2-3: Drying Supernatant in Graduated Cylinders

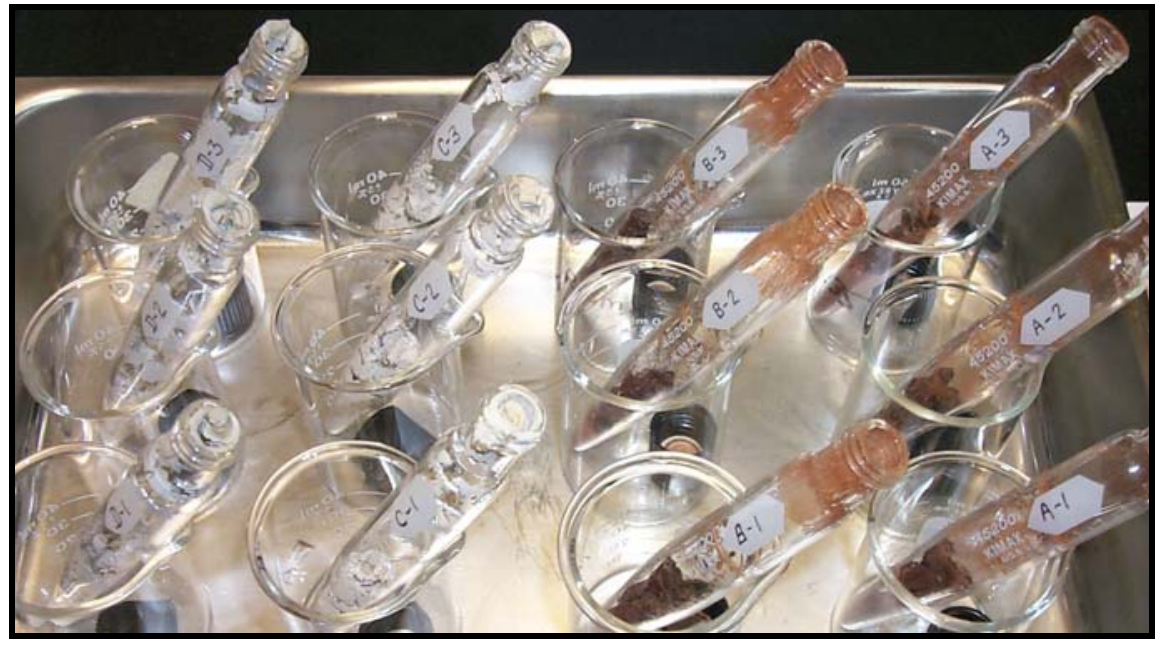

Figure 2-4: Dried Centrifuged Solids in Cones

Generally, these methods are not designed for production plant operations and are not recommended for implementation at WTP. The Defense Waste Processing Facility (DWPF) uses microwave ovens to obtain solids analysis. Additionally, infrared (heating element) drying ovens have also been tested on WTP slurries and have provided accurate and reliable results in a fraction of the time it takes to conduct the analysis compared to the method used in this study.

Each FIU QARD and clay sample was run in triplicate, with the averaged results reported in Table 1-2. 


\subsubsection{Density and Wt\% Total Solids - Laponite}

The density of the Laponite sample was measured by placing a 7 to $10 \mathrm{~mL}$ sample into a centrifuge cone and weighing the cone. The sample was then placed into a centrifuge and centrifuged for 1 hour at 1000 gravities. The volume of centrifuged product was then read and the density calculated. After centrifugation, there were no air bubbles in the Laponite samples.

The wt $\%$ total solids were determined by placing approximately a 10 gram sample of the Laponite onto a plate and the initial mass of Laponite recorded. The sample was then placed into an oven at $90^{\circ} \mathrm{C}$ for 24 hours, the temperature of the oven was increased to $105^{\circ} \mathrm{C}$ and the sample was allowed to dry up to 2 days or until the dry mass stabilized. The wt $\%$ total solids were determined by dividing the dry mass by the initial mass and multiplying this ratio by $100 \%$.

Each FIU Laponite sample was run in triplicate, with the averaged results reported in Table 1-2.

\subsubsection{Settling Results of the FIU Samples}

Settling tests were preformed on the clay and QARD AZ-101 samples as described in 24590WTP-GPG-RTD-001 [Ref. 1]. Each sample was placed into a graduated centrifuging cone (to be used for the density, wt $\%$ and vol\% determinations after the completion of the settling test), shaken to homogenize the sample, and allowed to settle for at least three days. Three sub-samples from each slurry sample were homogenized within 10 seconds of each other and placed into a centrifuge cone tube holder. Volume measurements of the interface layer, which is the layer between the clear supernatant and settling slurry, and the elapsed settling times were recorded. Figure 2-1 shows the graduated centrifuge tubes used for the settling test.

The WTP-QARD-112803-Feed-1 sample was the only sample that yielded observable settling data. The other QARD samples and all of the clay samples adhered to the centrifuge cone inner wall upon shaking, such that if an interface layer was generated, it could not be visually observed. Fissures formed in the adhered inner wall coating after several hours (Figure 2-1); however the actual solids/supernatant interface could not be determined. The normalized interface level was then determined by dividing the volume of settled solids by the total volume. The average normalized interface level of the three WTP-QARD-112803Feed-1 samples is reported in Table 1-3 and shown in Figure 2-5. 
The settling data has not been corrected for wall or hindered settling effects. The use of this data is limited only to decanting operations or to determine the amount of settled solids. The data can also be used to determine if rheological measurements using standardized rotational bench scale rheometers can be utilized to measure the rheological properties of the sample based on how quickly the interface is produced relative to the time it takes to perform the flow curve measurements. There are established standards for settling tests that are used for decanting, but the measuring vessel and sample size are much greater than what is used here. The larger vessel size is used to minimize wall effects.

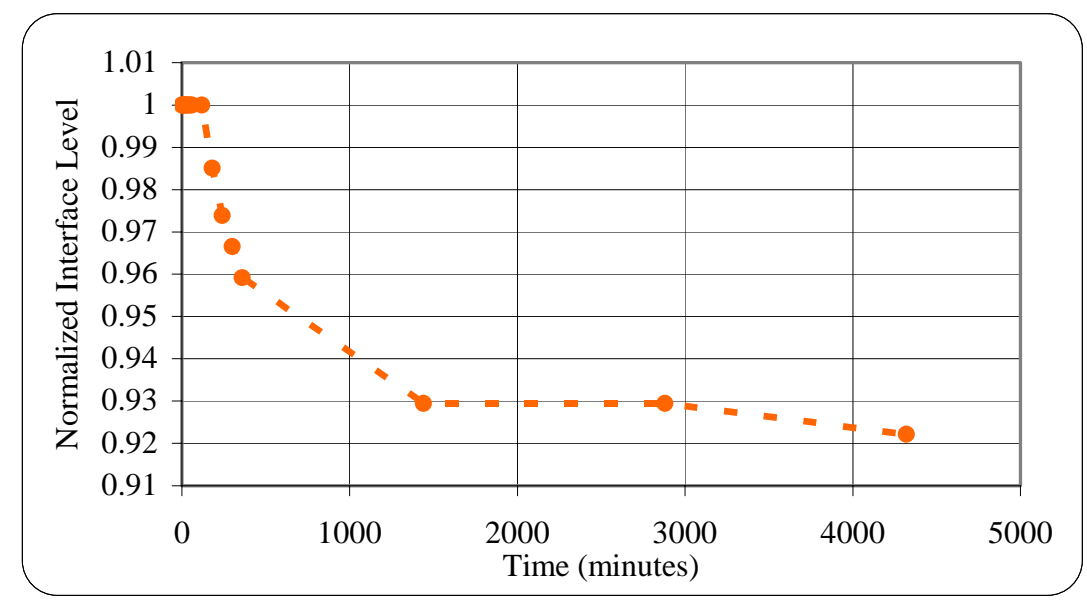

Figure 2-5: Normalized Settling Curves For FIU WTP-QARD-112803-Feed Sample

\subsection{RHEOLOGY MEASUREMENT}

\subsubsection{Instrumentation}

The Haake RS150 and RS600 rheometers were used for all rheological measurements in this task. The RS150 and RS600 were used for flow curve measurements and the RS150 used for vane measurements. The RS150 and RS600 specifications are shown in Table 2-2.

Table 2-2: Specifications of the RS150 and RS600

\begin{tabular}{|c|c|c|c|}
\hline Specification & Units & RS150 & RS600 \\
\hline Maximum Torque & N-m & 0.15 & 0.20 \\
\hline Minimum Torque & N-m & $0.5 \mathrm{E}-7$ & $0.5 \mathrm{E}-7$ \\
\hline Maximum Speed & RPM & 1200 & 1500 \\
\hline Minimum Speed & RPM & 0.01 & 0.001 \\
\hline
\end{tabular}




\subsubsection{Flow Curve Measurements Using Concentric Geometry}

Flow curve measurements were obtained using the Z41 cylindrical rotor. The Z41 rotor specifications are shown in Table 2-3. The bob and its cup are initially installed onto the rheometer. The zero reference point (point at which the Z41 rotor makes full contact with the bottom of the cup) is then determined by the rheometer. The Z41 rotor and cup are removed and approximately $15 \mathrm{~mL}$ of sample is added to the cup. The cup and Z41 rotor are then reinstalled onto the rheometer. The rheometer drives the Z41 rotor into the predetermined bottom off-set position of 3-mm and excess sample is trimmed from the bob. A cooling/heating bath is used to control the temperature of the rotor/sample/cup at $25^{\circ} \mathrm{C}$. The rheometers are programmed to control the rate at which the rotor spins and measures both the rotational speed and the torque (the resistance to shear). The shear stress at the wall of the rotating rotor is then calculated (internally by the Haake ${ }^{\mathrm{TM}}$ software) based on the product of the measured torque and geometry (A-factor) of the bob. The shear rate of the rotating rotor is calculated as the product of the measured speed and geometry (this M-factor assumes the fluid is Newtonian) of the rotor. The A-factor, M-factor, shear rate range and the linear ramp up time, hold time at maximum shear rate, and linear ramp down time are provided in Table 2-3. The linear ramp rates (or acceleration) is $\pm 200 \mathrm{sec}^{-1}$ per minute. 
Table 2-3: Z41 Rotor Specification and Program Ramp Rates

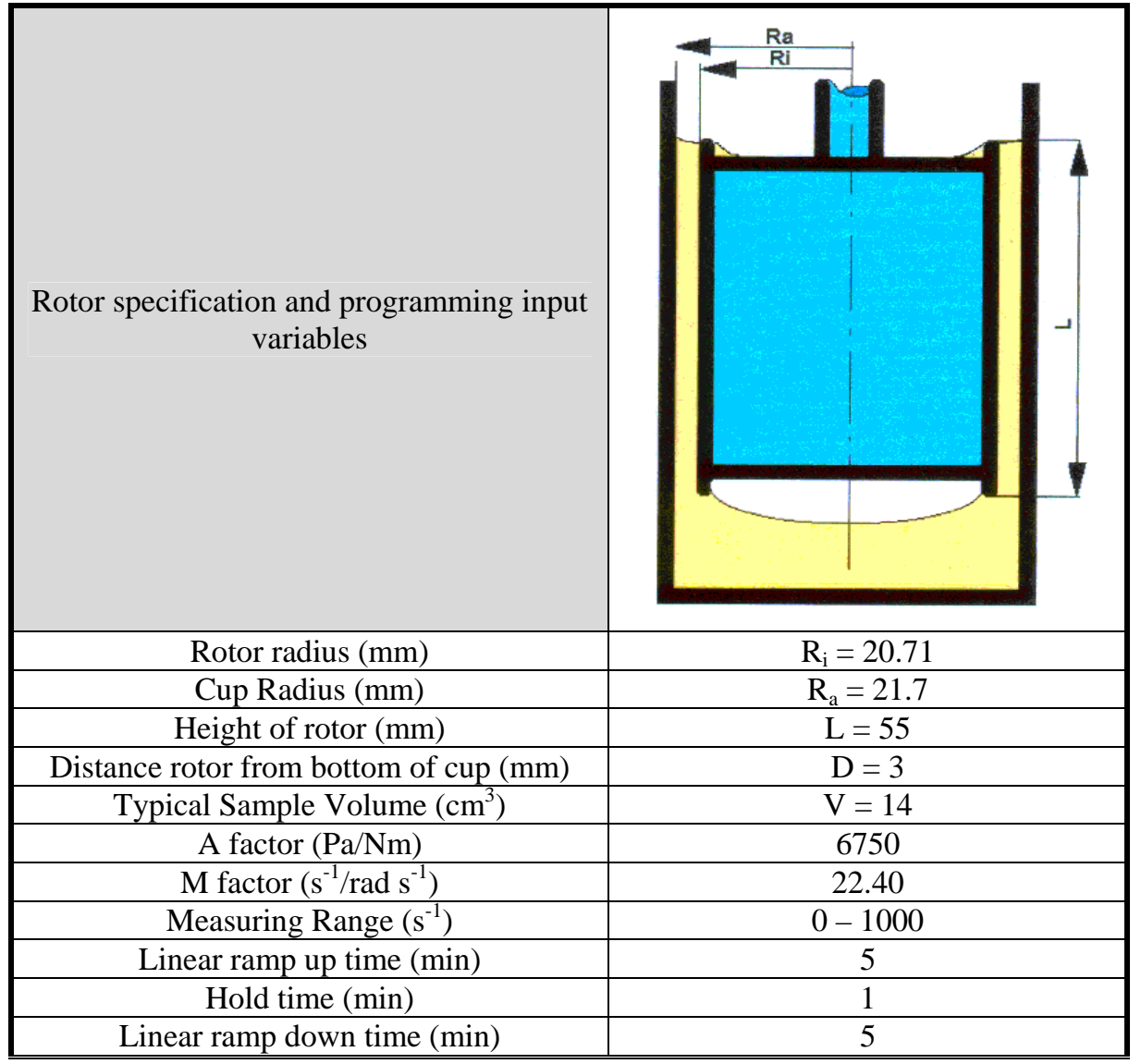

Prior to any flow curve measurement, the rotor and cup are inspected for visual damage that could potentially impact the rheological measurement. A National Institute of Standards and Technology (NIST) traceable Newtonian oil standard is then used to verify the operability of the rheometer at a measurement temperature of $25^{\circ} \mathrm{C}$. The resulting flow curves are analyzed as a Newtonian fluid and the calculated viscosity is compared to that of the NIST traceable Newtonian oil standard. The rheometer is considered operable if the calculated viscosity is within $\pm 10 \%$ of the NIST traceable Newtonian oil standard viscosity. The NIST traceable Newtonian oil standard was run each day when flow curve measurements were performed. This met the QA requirements in verifying the operability of the rheometer using a NIST traceable Newtonian oil standard as stated in reference 1. 
Upon completion of a flow curve measurement, the bottom of the Z41 rotor is inspected to determine if the sample completely filled the void space at the bottom of the Z41 rotor. This void space is an air buffer, where the shear stress contribution from the air is negligible compared to the shear stress from the cylindrical section of the Z41 rotor which are in full contact with the fluid during the flow curve measurement. If the void space is completely filled with the sample, after the measurement is completed, then a fresh sample is loaded and the Z41 rotor inserted into the sample at a slower insertion rate. This process is repeated until there is evidence that the sample is not impacting the measurement due to bottom void space being completely filled. If this void space is completely filled, the resulting flow curve is more viscous.

\subsubsection{Shear Strength Measurement Using Vane Geometry}

The RS150 was used to perform the vane measurements. The RS150 was functionally verified using the Z41 and a NIST traceable Newtonian oil standard within the frequency as specified in reference 1 .

Vanes have been used [Ref. 4 through 17] to measure the yield stress of non-Newtonian fluids as shown in Figure 2-6. The vane is inserted into the fluid and rotated at a very slow speed. The surface area used to determine the shear stress is the surface area produced by the vane, which is cylindrical. It has been shown that this is a good assumption [Ref. 5, 6, 9, and 10] for determining the yield stress of the fluid as the vane slowly rotates through it. The derived equation (3.3) assumes the stress is constant on all surfaces. The shearing due to the immersed section of the vane shaft, stress contribution of the immersed section of the shaft, and the wall effects are negligible when meeting the criteria as shown in Figure 2-6. The length of immersed shaft will need to be considered if it length starts to impact the measured stress. The exclusion of the shear stress contribution of the immersed shaft length overestimates the shear stress.

$$
\begin{aligned}
& \tau=\frac{\Gamma}{\frac{\pi \cdot D^{3}}{2}\left(\frac{H}{D}+\frac{1}{3}\right)}=A \cdot \Gamma \\
& \text { Where } \Gamma=\text { measured torque }(\mathrm{N}-\mathrm{m} \text { or } \% \text { torque }) \\
& \mathrm{D}=\text { diameter of vane }(\mathrm{m}) \\
& \mathrm{H}=\text { height of vane }(\mathrm{m}) \\
& \mathrm{A}=\text { geometric constant }\left(\mathrm{m}^{3} \text { or } \mathrm{Pa} / \% \Gamma\right) \\
& \tau=\text { shear stress }(\mathrm{Pa})
\end{aligned}
$$


WSRC-TR-2004-00010, REV. 0

SRT-RPP-2004-00001, REV. 0

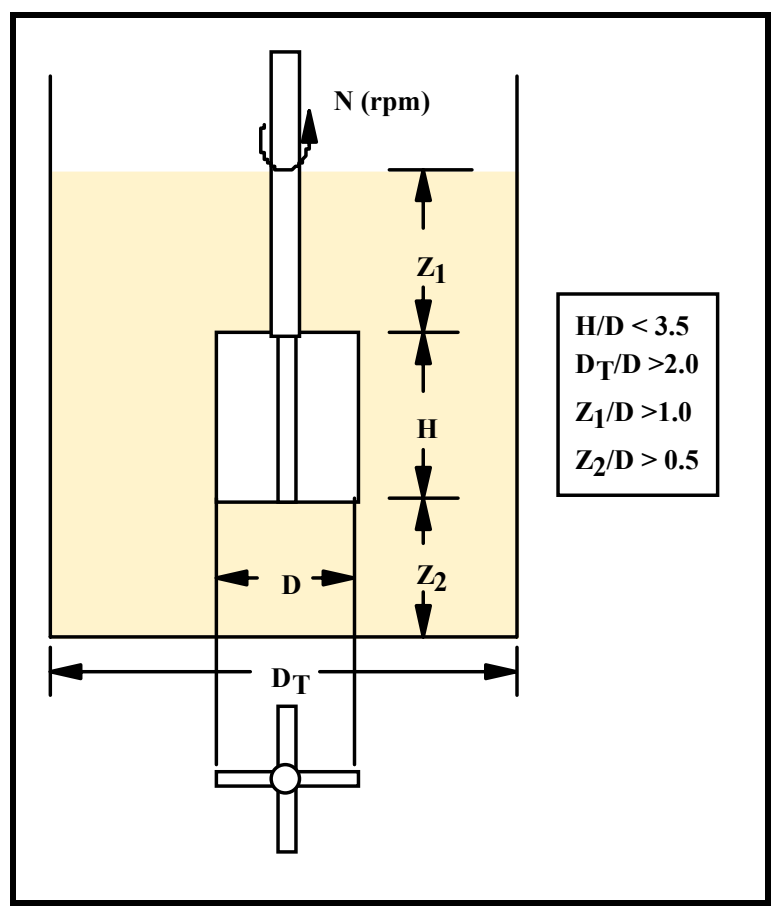

Figure 2-6: Vane Geometric Requirements

A typical stress versus time (or displacement) curve is shown in Figure 2-7. The initial response for a non-Newtonian fluid having a yield stress is typically linear and this slope is called the Hookean elastic modulus $(\mathrm{G})$. The point of departure from this linear region is called the static yield stress [Ref. 4] when the fluid starts to transition from a fully elastic to viscoelastic behavior. At the maximum stress, the behavior of the material transitions between viscoelastic and fully viscous and is called the yield stress (also known as the dynamic yield stress). 
WSRC-TR-2004-00010, REV. 0

SRT-RPP-2004-00001, REV. 0

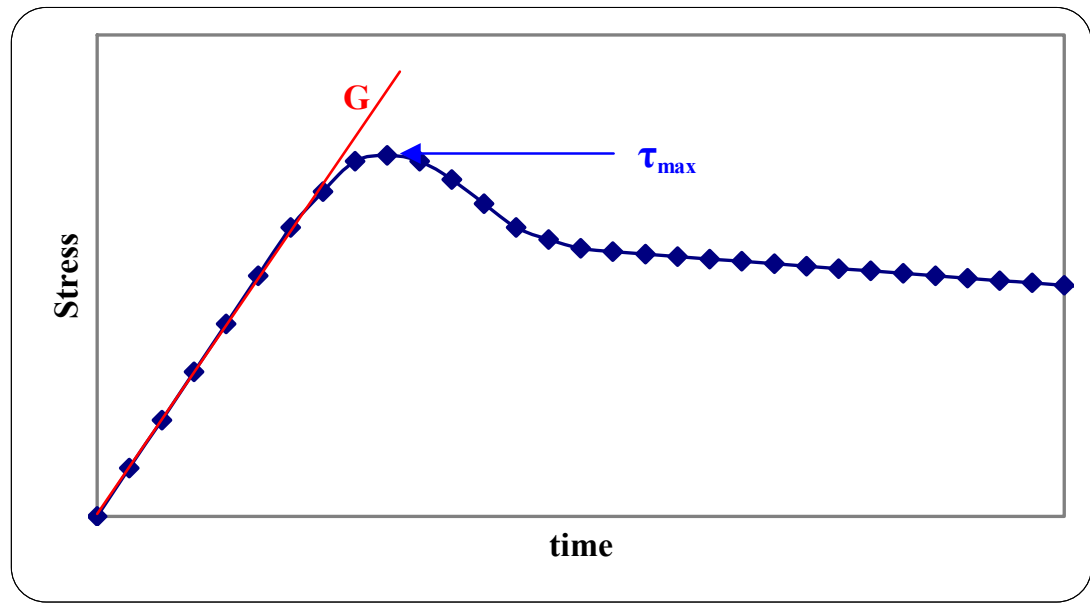

Figure 2-7: Typical Vane Torque versus Time/Displacement Curve

The vane dimensions used in this task were $\mathrm{D}=\mathrm{H}=16 \mathrm{~mm}$. The A factor for this vane was calculated and used in the RS150 to calculate the shear stress from the measured torque, given the measured torque is in $\mathrm{N}-\mathrm{m}$. The A factor used is shown in equation 3.4.

$$
A=\frac{A}{N \cdot m}=\frac{2}{\pi \cdot(0.016 m)^{3} \cdot\left(\left(\frac{16}{16}\right)+\frac{1}{3}\right)} \cdot \frac{N \cdot m}{N \cdot m} \cdot \frac{P a}{\frac{N}{m^{2}}}=116,568 \frac{P a}{N \cdot m}
$$

The $\mathrm{M}$ factor for this vane was set at $1.0 \mathrm{sec}^{-1} /\left(\mathrm{rad} \cdot \mathrm{s}^{-1}\right)$. Going through the same exercise as that shown in equation 3.4, for a rotational speed of 0.3 RPM, the controlled shear rate was $0.03 \mathrm{sec}^{-1}$. The rotational speed was also visually verified at approximately 0.3 revolutions per minute (RPM). This rotational speed is recommended in 24590-WTP-GPG-RTD-001 [Ref. 1].

The WTP terminology for yield stress using the vane method is shear strength.

\subsubsection{Flow Curve Results}

Duplicate flow curves of each FIU fluid were measured at $25^{\circ} \mathrm{C}$. The FIU samples were homogenized, using care to avoid the introduction of air into the sample, and immediately transferred to the cup. The Z41 was inserted into the cup, excess sample trimmed, and the measurement performed. During these measurements, settling was not an issue. For the WTP-Clay-121603-Used FIU-HCET dilute clay sample, the insertion speed of the Z41 into the sample had to be reduced due to the sample completely filling the void space at the bottom of the Z41. 
The following non-Newtonian rheological models (equation 3.5, 3.6 and 3.7) were used to fit the down flow curves of each of the FIU fluids. The down flow curves were fitted from 0 to $1000 \mathrm{sec}^{-1}$ using the Haake ${ }^{\mathrm{TM}}$ software and the results are shown in

Table 1-5. Individual flow curves are provided in Appendix A.

Power Law:

$$
\tau=m \cdot \dot{\gamma}^{n}
$$

Where: $\mathrm{m}=$ the consistency coefficient $\left(\mathrm{Pa}-\mathrm{sec}^{\mathrm{n}}\right)$

$\mathrm{n}=$ the power law exponent (unit less)

$\dot{\gamma}=$ shear rate $\left(\mathrm{sec}^{-1}\right)$

Bingham Plastic:

$\tau=\tau_{B P}+\eta_{B P} \cdot \dot{\gamma}$

Where: $\quad \tau_{\mathrm{BP}}=$ Bingham Plastic yield stress $(\mathrm{Pa})$

$\eta_{\mathrm{BP}}=$ Bingham Plastic Viscosity (or consistency) (cP)

Herschel-Bulkley:

$\tau=\tau_{H B}+k \cdot \dot{\gamma}^{b}$

Where: $\tau_{\mathrm{HB}}=$ Herschel-Bulkley yield stress $(\mathrm{Pa})$

$\mathrm{k}=$ the consistency coefficient $\left(\mathrm{Pa}-\mathrm{Sec}^{\mathrm{n}}\right)$

$\mathrm{b}=$ the power law exponent (unit less)

\subsubsection{Vane Shear Strength Results}

Each FIU sample was homogenized and approximately $400 \mathrm{~mL}$ of the fluid was transferred to a $400 \mathrm{~mL}$ sample cup that permitted four independent measurements that met the criteria stated in Figure 2-6. The $400 \mathrm{~mL}$ sample cups were placed into an oven at $25^{\circ} \mathrm{C}$ for the duration of the vane shear strength measurements and were only removed from the oven during the actual measurement. The four measurement positions on the $400 \mathrm{~mL}$ sample cup were determined by marking the outside of the cup 90 degrees apart and permitting only one measurement at each location. Duplicate measurements were performed for each measurement time of settling. Hence, only two measurement times of settling could be performed on the $400 \mathrm{~mL}$ sample, after which the sample cup was re-homogenized by capping it and vigorously shaking the contents in the cup. This cycle of measuring and rehomogenization was maintained until all the settling times as specified in Table 1-1 were performed. For the initial homogenized condition at $\mathrm{t}=0$, the sample was mixed and immediately measured.

The averaged vane results for each FIU sample are shown in Table 1-4 and are provided in graphical form in Appendix B. 
In Table 1-4, the shear strength of the WTP-QARD-112803-Feed increased drastically after two days of settling. This was visually confirmed after the vane was removed from the sample after the 48 hour measurement, where the fluid stuck to the vane as a clump as shown in Figure 2-8. Additionally, it was observed that when this sample was received from FIU and the contents transferred from the original small mouth $500 \mathrm{~mL}$ bottle to a wide mouth $500 \mathrm{~mL}$ bottle, a large clump of material was still present in the original $500 \mathrm{~mL}$ bottle. The clump in the original $500 \mathrm{~mL}$ bottle was transferred to the $500 \mathrm{~mL}$ wide mouth bottle prior to any physical property measurement.

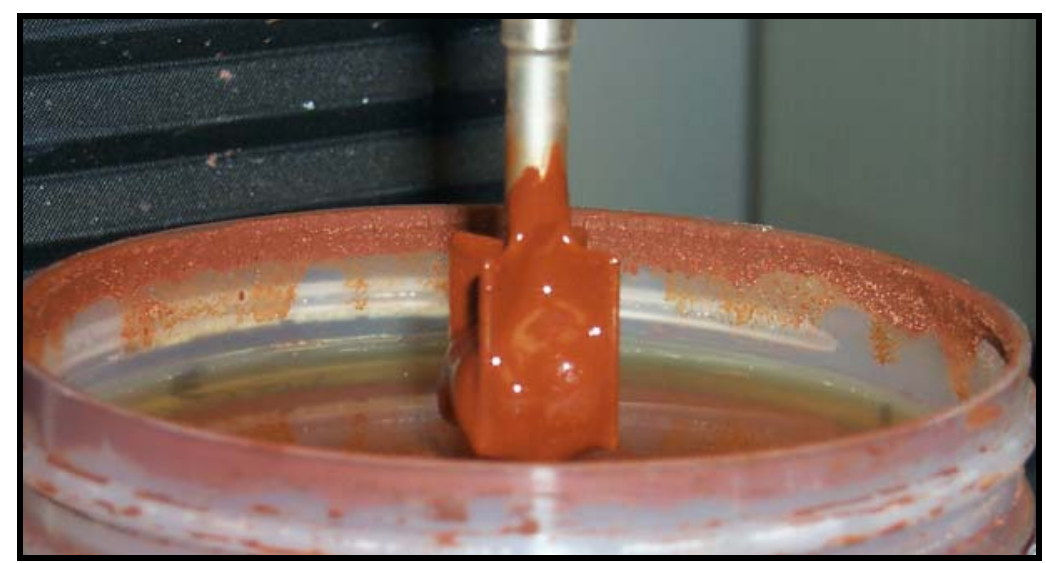

Figure 2-8: FIU WTP-QARD-112803-Feed Vane 48 hour Measurement

Table 2-4 is a comparison of the yield stress (as determined from the flow curve using the rheological models that have a yield stress property) to that of the vane shear strength (of the homogenized fluids at $t=0$ ). In all cases, the Herschel-Bulkely predicts a lower yield stress than either the Bingham Plastic or vane shear strengths. The results between the Bingham Plastic yield stress and vane shear strength are comparable for the QARD and initial clay samples. In case of the Laponite samples, the beginning part of their flow curves (see Figure A - 5 and Figure A - 6) have a pronounced power law behavior, thus the Bingham Plastic model over estimated the yield stress. 
WSRC-TR-2004-00010, REV. 0

SRT-RPP-2004-00001, REV. 0

Table 2-4: Yield Stress Comparison

\begin{tabular}{|c|c|c|c|}
\hline \multirow[b]{2}{*}{ FIU Sample Description } & \multicolumn{3}{|c|}{ Yield Stress $(\mathrm{Pa})$} \\
\hline & $\begin{array}{c}\text { Bingham Plastic } \\
\left(\tau_{\mathrm{BP}}\right)\end{array}$ & $\begin{array}{l}\text { Herschel-Bulkley } \\
\left(\tau_{\mathrm{HB}}\right)\end{array}$ & $\begin{array}{c}\text { Vane } t=0 \\
\left(\tau_{\text {VANE }}\right)\end{array}$ \\
\hline WTP-1B (Laponite) Date 11/24/03 Feed & 7.6 & 4.5 & 16.4 \\
\hline WTP-1B (Laponite) Date 11/24/03 Used & 7.6 & 4.3 & 17.8 \\
\hline WTP-QARD-112803-Feed & 3.4 & 2.4 & 3.3 \\
\hline WTP-QARD-113003-Used & 16.4 & 13.1 & 18.6 \\
\hline $\begin{array}{l}\text { 27\% Clay Slurry New From Tote WTP- } \\
\text { Clay } 1-12 / 6-1\end{array}$ & 16.2 & 13.2 & 19.7 \\
\hline $\begin{array}{c}\text { 27\% Clay Slurry Used Following Ar + } \\
\text { H2O2 Tests, WTP-Clay1-12/6-2 }\end{array}$ & 12.0 & 8.9 & 13.7 \\
\hline $\begin{array}{c}\text { Diluted Clay To Mimic QARD Rheology } \\
\text { New, WTP-Clay2-12/6-1 }\end{array}$ & 7.1 & 4.6 & 8.3 \\
\hline $\begin{array}{l}\text { Diluted Clay After } \mathrm{H} 2 \mathrm{O} 2 \text { Injection \& } \\
\text { Release Rheology New, WTP-Clay2- } \\
\qquad 12 / 6-1\end{array}$ & 5.2 & 3.1 & 6.1 \\
\hline $\begin{array}{l}\text { WTP-Clay-121603-Used1 FIU-HCET } \\
\text { Diluted Clay }\end{array}$ & 31.1 & 11.4 & 17.0 \\
\hline $\begin{array}{l}\text { WTP-QARD-12/7-1 After } 2^{\text {nd }} \mathrm{H} 2 \mathrm{O} 2 \\
\text { Injection and Release }\end{array}$ & 14.0 & 11.3 & 14.3 \\
\hline $\begin{array}{l}\text { WTP-QARD-12/8-2 After } 3^{\text {rd }} \mathrm{H} 2 \mathrm{O} 2 \\
\text { Injection and Release }\end{array}$ & 13.5 & 10.7 & 13.7 \\
\hline
\end{tabular}

\section{3 pH MEASUREMENT}

The $\mathrm{pH}$, though not required, was measured, since the use of hydrogen peroxide affects the $\mathrm{pH}$ of the resulting slurries. $\mathrm{pH}$ changes are known to affect the rheology of slurries.

The $\mathrm{pHs}$ of the FIU samples were measured using an IQ Scientific Instruments $\mathrm{pH}$ meter fitted with an IQ Scientific Instruments stainless steel electrode reference $\mathrm{pH}$ probe at ambient conditions. The functionality of the $\mathrm{pH}$ meter and probe were confirmed by

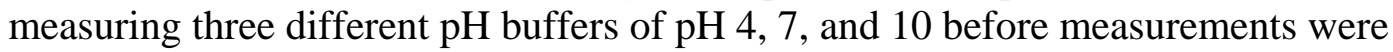
performed. These Fisher Scientific $\mathrm{pH}$ buffers are certified to within $+/-0.02 \mathrm{pH}$ units and were used within the designated expiration date listed on each buffer.

The results are shown in Table 1-2. The pH of the final clay product (121603-Used1 FIUHCET Diluted Clay) was very acidic as compared to the others. 
WSRC-TR-2004-00010, REV. 0

SRT-RPP-2004-00001, REV. 0

\subsection{ELEMENTAL COMPOSITION}

Elemental analyses of the samples shipped to or received from FIU were not specified in reference 2 .

The elemental composition of the QARD AZ-101 simulant, prior to any processing at FIU is shown in Table 2-5. This elemental composition was taken directly from reference 18 . The method in how the QARD AZ-101 simulants were made for FIU is specified in Appendix F of reference 18 .

Table 2-5: Elemental Composition of QARD AZ-101

\begin{tabular}{|c|c|c|c|}
\hline Species & $\boldsymbol{\mu g} / \mathbf{g r a m}$ solids & Species & $\boldsymbol{\mu}$ g/gram solids \\
\hline $\mathrm{Ag}$ & $<280$ & $\mathrm{Mn}$ & 5438 \\
\hline $\mathrm{Al}$ & 86659 & $\mathrm{Mo}$ & $<90$ \\
\hline $\mathrm{B}$ & 3573 & $\mathrm{Na}$ & 42212 \\
\hline $\mathrm{Ba}$ & 1657 & $\mathrm{Nd}$ & 3108 \\
\hline $\mathrm{C}_{2} \mathrm{O}_{4}$ & 186 & $\mathrm{Ni}$ & 9970 \\
\hline $\mathrm{Ca}$ & 8158 & $\mathrm{NO}_{2}$ & 4623 \\
\hline $\mathrm{Cd}$ & 11265 & $\mathrm{NO}_{3}$ & 48686 \\
\hline $\mathrm{Ce}$ & 3444 & $\mathrm{P}$ & 2564 \\
\hline $\mathrm{Cl}$ & 443 & $\mathrm{PO}_{4}$ & 627 \\
\hline $\mathrm{Co}$ & 150 & $\mathrm{Rh}$ & 546 \\
\hline $\mathrm{Cr}$ & 2344 & $\mathrm{Ru}$ & 947 \\
\hline $\mathrm{Cu}$ & 609 & $\mathrm{Si}$ & 15794 \\
\hline $\mathrm{F}$ & 172 & $\mathrm{Sn}$ & 1554 \\
\hline $\mathrm{Fe}$ & 202384 & $\mathrm{SO}$ & 1997 \\
\hline $\mathrm{K}$ & 3172 & $\mathrm{Ti}$ & 341 \\
\hline $\mathrm{K}$ & 2508 & $\mathrm{Zn}$ & 337 \\
\hline $\mathrm{La}$ & 3755 & $\mathrm{Zr}$ & 61505 \\
\hline $\mathrm{Mg}$ & 1554 & & \\
\hline
\end{tabular}

The elemental composition of the Laponite and clay simulants, which were provided directly from WTP R\&T to FIU were not analyzed. 
WSRC-TR-2004-00010, REV. 0

SRT-RPP-2004-00001, REV. 0

This page intentionally left blank. 
WSRC-TR-2004-00010, REV. 0

SRT-RPP-2004-00001, REV. 0

\subsection{REFERENCES}

1. Smith, G.L., and Prindiville, K., Guidelines for Performing Chemical, Physical, and Rheological Properties Measurements, 24590-WTP-GPG-RTD-001, Rev. 0, May 20, 2002.

2. CNN 066794, NTP with SRTC Simulant Rheology Measurements for FIU, Rev.1, November 11, 2003.

3. Hansen, E. K., "Task Technical and QA Plan - Physical Characterization of FIU Simulants", WSRC-TR-2003-00538, Rev. 0, 2003

4. Liddell, P.V. and Boger D.V., "Yield Stress Measurements with the vane", Journal of Non-Newtonian Fluid Mechanics, Vol. 63, pp 235-261, 1996

5. Nguyen, Q.D. and Boger D.V., "Yield Stress Measurements for Concentrated Suspensions", Journal of Rheology, Vol. 27, pp 321-349, 1983

6. Nguyen, Q.D. and Boger D.V., "Direct Yield Stress Measurement with the Vane Method", Journal of Rheology", Vol. 29, pp 335-347, 1985

7. Barnes, H.A. and Nguyen, Q.D., "Review Rotating vane rheometry - a review", Journal of Non-Newtonian Fluid Mechanics, Vol. 98, pp 1-14, 2001

8. Saak, A.W., Jennings, H.M., and Shah S.P., "The influence of wall slip on yield stress and viscoelastic measurements of cement paste", Cement and Concrete Research, Vol. 31, pp 205-212, 2001

9. Barnes, H. A. and Carnali, J. O., "The vane-in-cup as a novel rheometer geometry for shear thinning and thixotropic materials", Journal of Rheology, Vol. 34, pp 841-866, 1990

10. Yoshimura, A. S., and Prud'Homme, P.K., “A Comparison of Techniques for Measuring Yield Stresses”, Journal of Rheology, Vol. 31, pp 699-710, 1987

11. Alderman, N. J., Meeten, G. H., and Sherwood, J. D., "Vane rheometry of bentonite gel”, Journal of Non-Newtonian Fluid Mechanics, Vol. 91, pp 291-310, 1991

12. Mohseni, M., Kautola, H., and Allen D.G., "The Viscoelastic Nature of Filamentous Fermentation Broths and Its Influence on the Directly Measured Yield Stress", Journal of Fermentation and Bioengineering, Vol. 83, pp 281-286, 1997

13. Keating, J. and Hannant, D. J., "The Effect of Rotation Rate on Gel Strength and Dynamic Yield Strength of Thixotropic Oil Well Cements Measured Using a Shear Vane", Journal of Rheology, Vol. 33, pp 1011-1020, 1989

14. Wardhaugh, L. T. and Boger, D. V., "The measurement and description of the yielding behavior of waxy crude oil", Journal of Rheology, Vol. 35, pp 1121-1165, 1991

15. Yan, J. and Jame, A.E., "The yield surface of viscoelastic and plastic fluids in a vane viscometer", Journal of Non-Newtonian Fluid Mechanics", Vol. 70, pp 237-253, 1997

16. Turian, R. M., Ma, T. W., Hsu, F.L.G., and Sung, D. J., "Characterization, settling, and rheology of concentrated fine particle mineral slurries", Power Technology, Vol. 93, pp. 219-233, 1997

17. Iyer, R.S. and Stanmore B., "The effect of water absorption and the role of fines on the yield stress of dense fly ash slurries", Cement and Concrete Research, Volume 29, pp 765-767, 1999 
WSRC-TR-2004-00010, REV. 0

SRT-RPP-2004-00001, REV. 0

18. Eibling, R. E., Schumacher, R. F., and Hansen, E. K., "Development of Simulants to Support Mixing Tests for High Level Waste and Low Activity Waste”, WSRC-TR2003-00220, Rev. 0, December 2003 
WSRC-TR-2004-00010, REV. 0

SRT-RPP-2004-00001, REV. 0

APPENDIX A - Flow Curves 
WSRC-TR-2004-00010, REV. 0

SRT-RPP-2004-00001, REV. 0

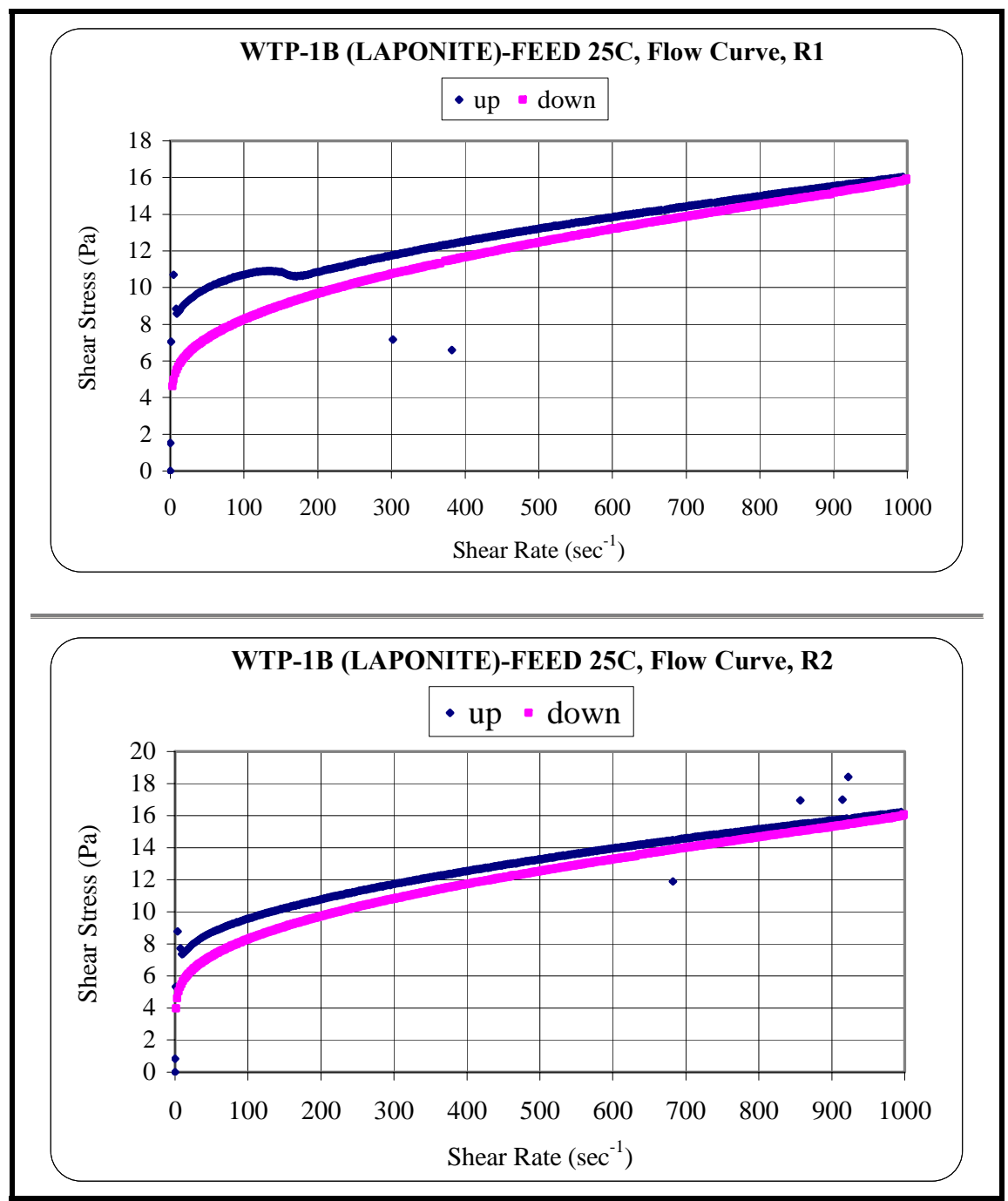

Figure A - 1: WTP-1B (Laponite) Date 11/24/03 Feed

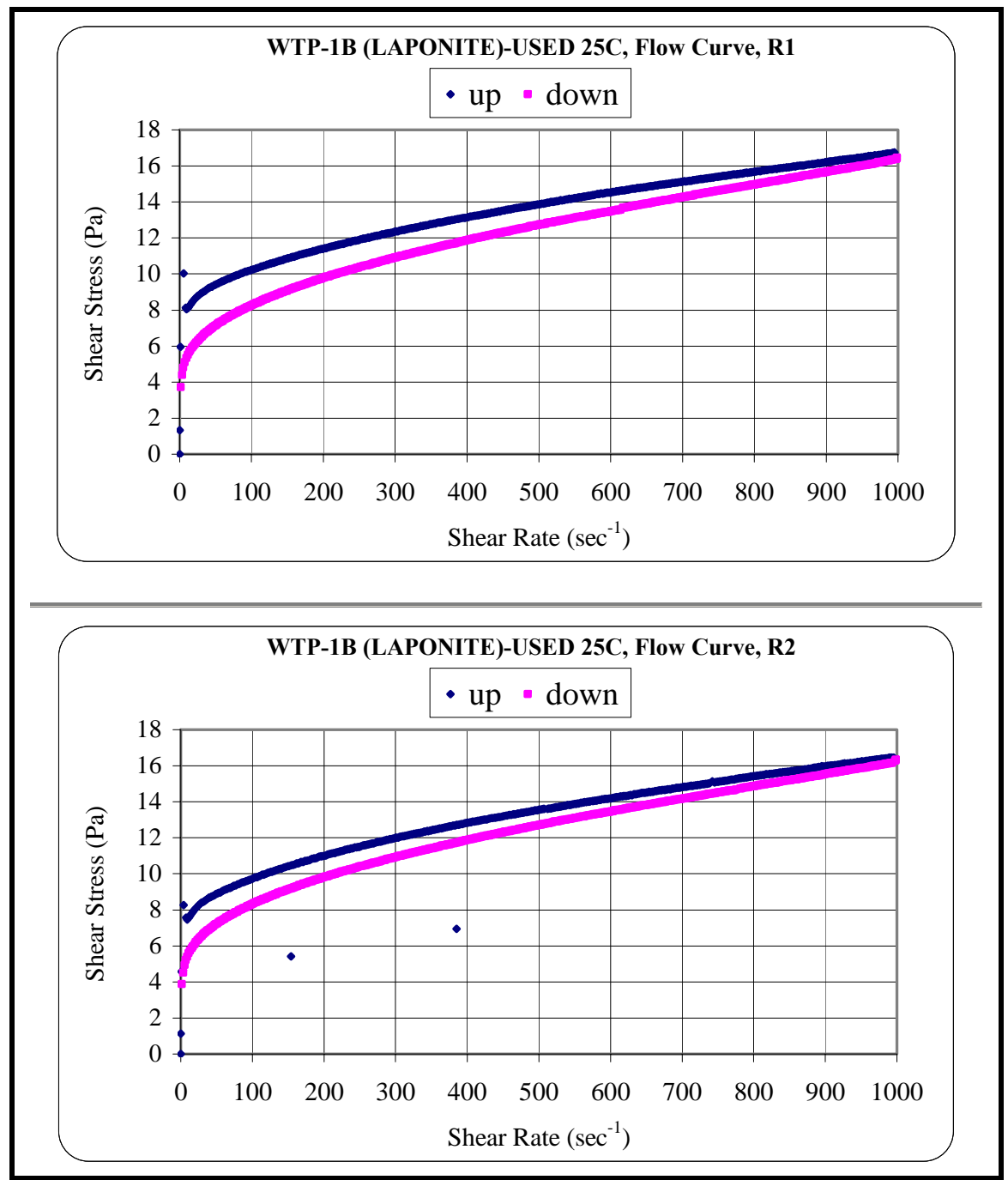

Figure A - 2: WTP-1B (Laponite) USED 
WSRC-TR-2004-00010, REV. 0 SRT-RPP-2004-00001, REV. 0

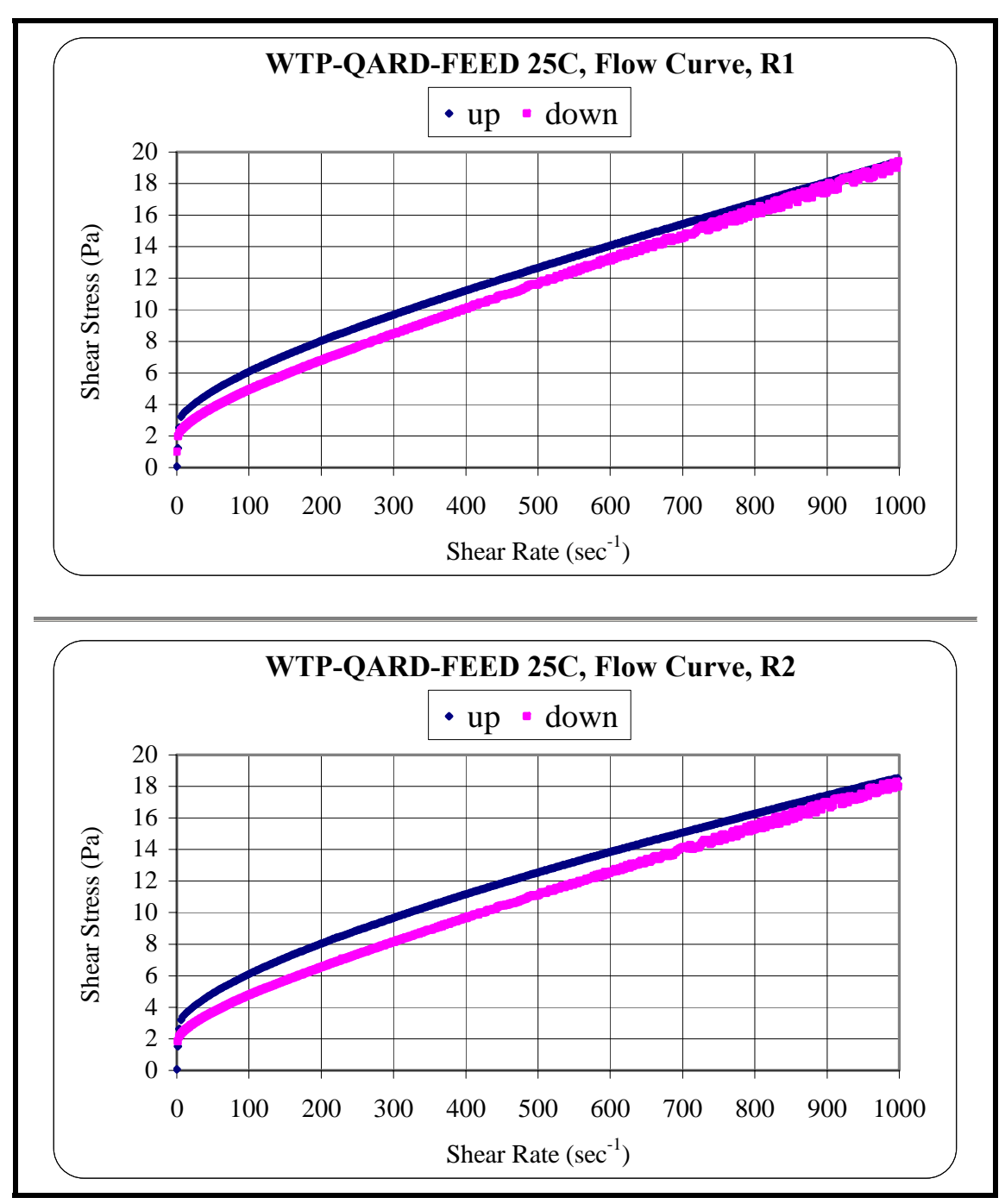

Figure A - 3: WTP-QARD-112803-Feed

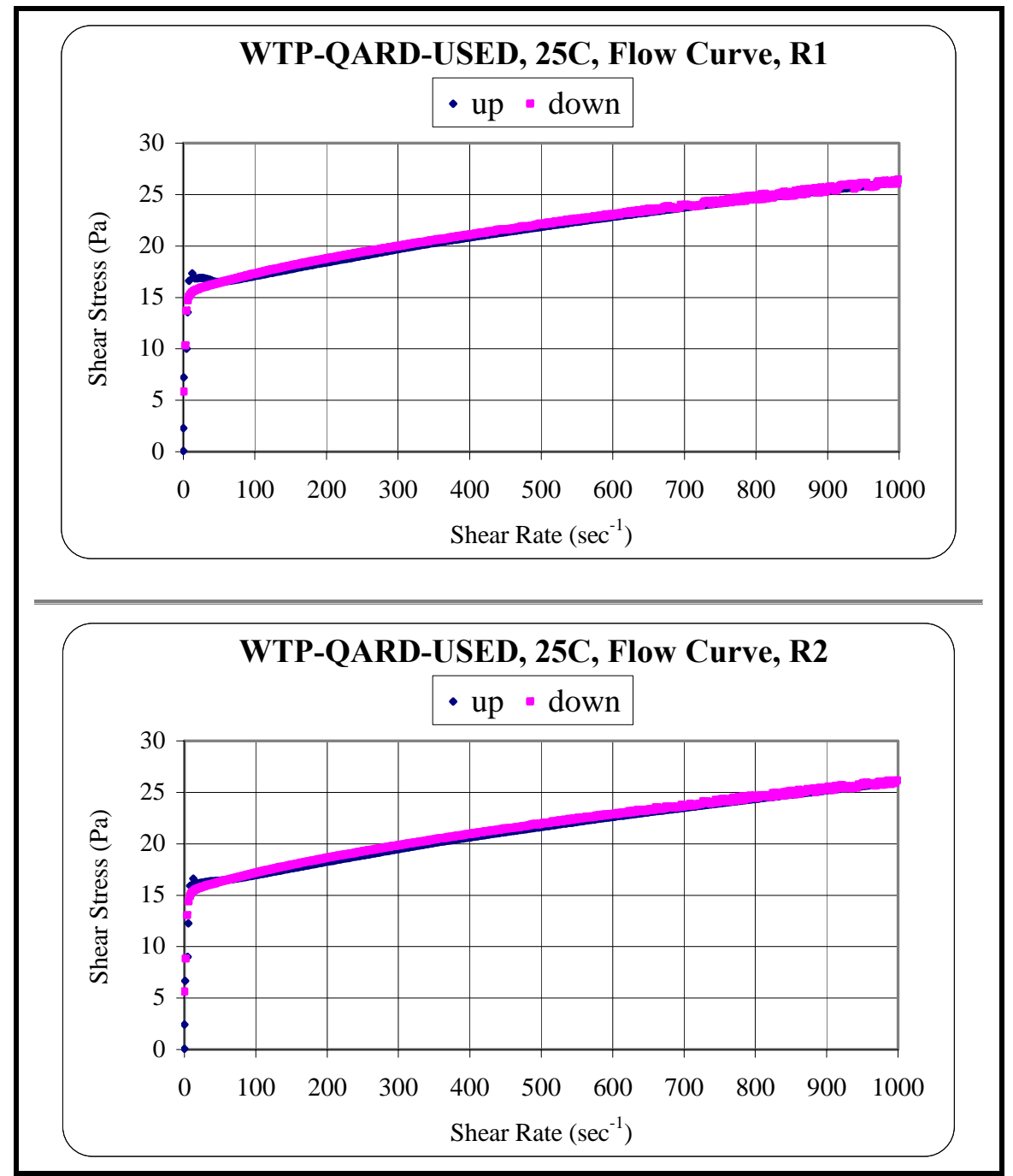

Figure A - 4: WTP-QARD-113003-USED 


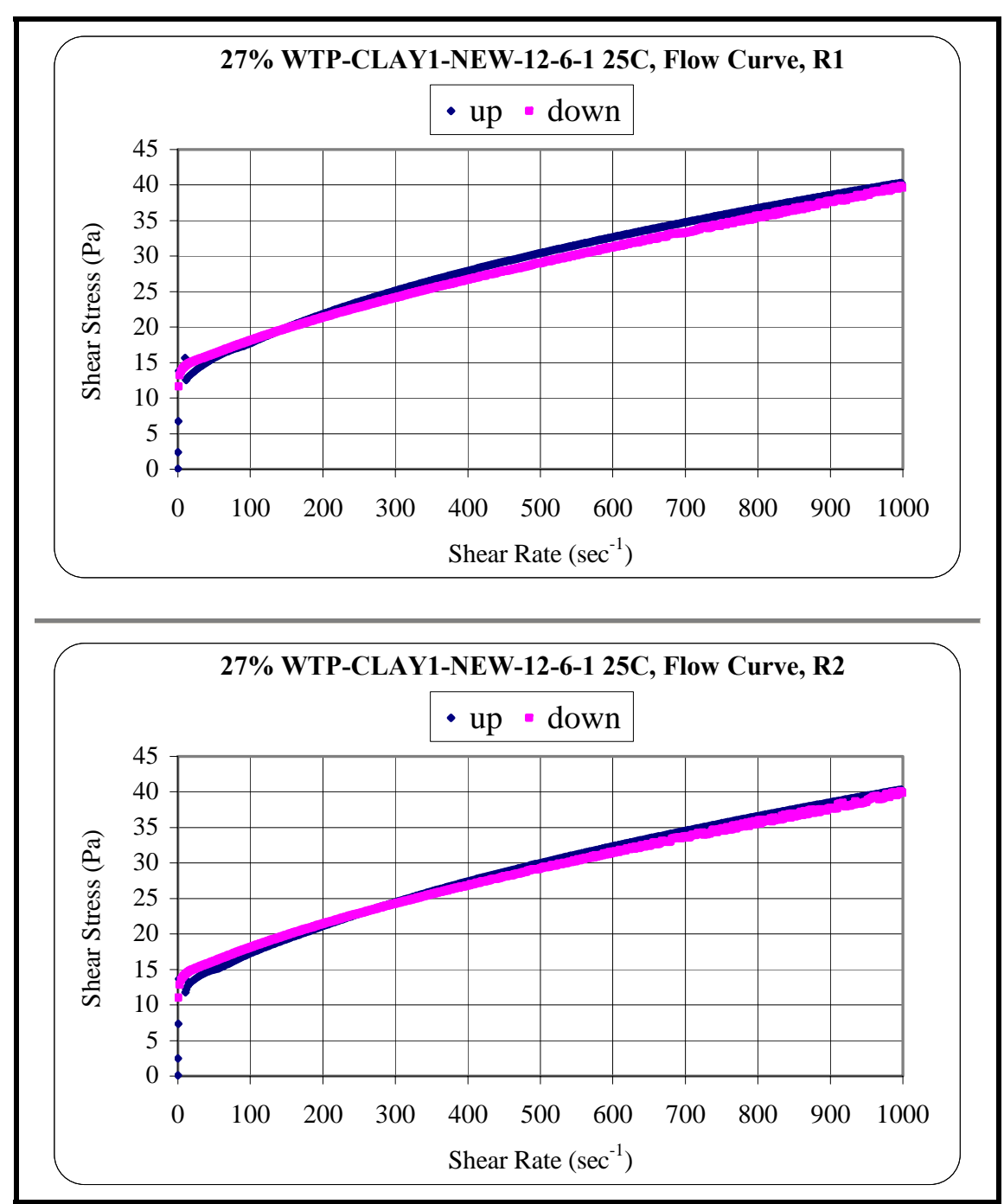

Figure A - 5: 27\% Clay Slurry From New Tote WTP Clay 1-12/6/-1

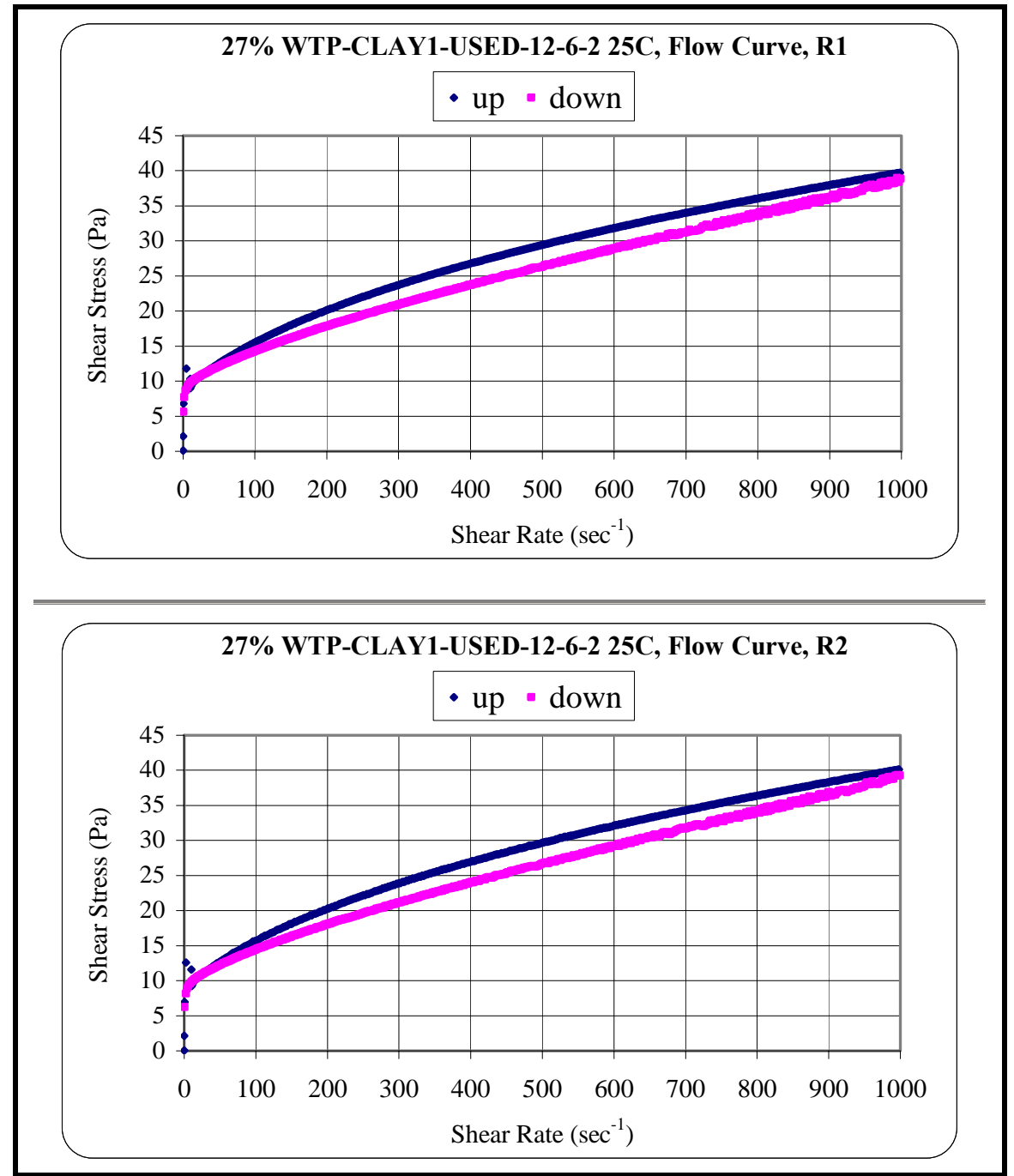

Figure A - 6: 27\% Clay Slurry Used Following Ar + H2O2 Tests, WTP-Clay 1-12/6-1 


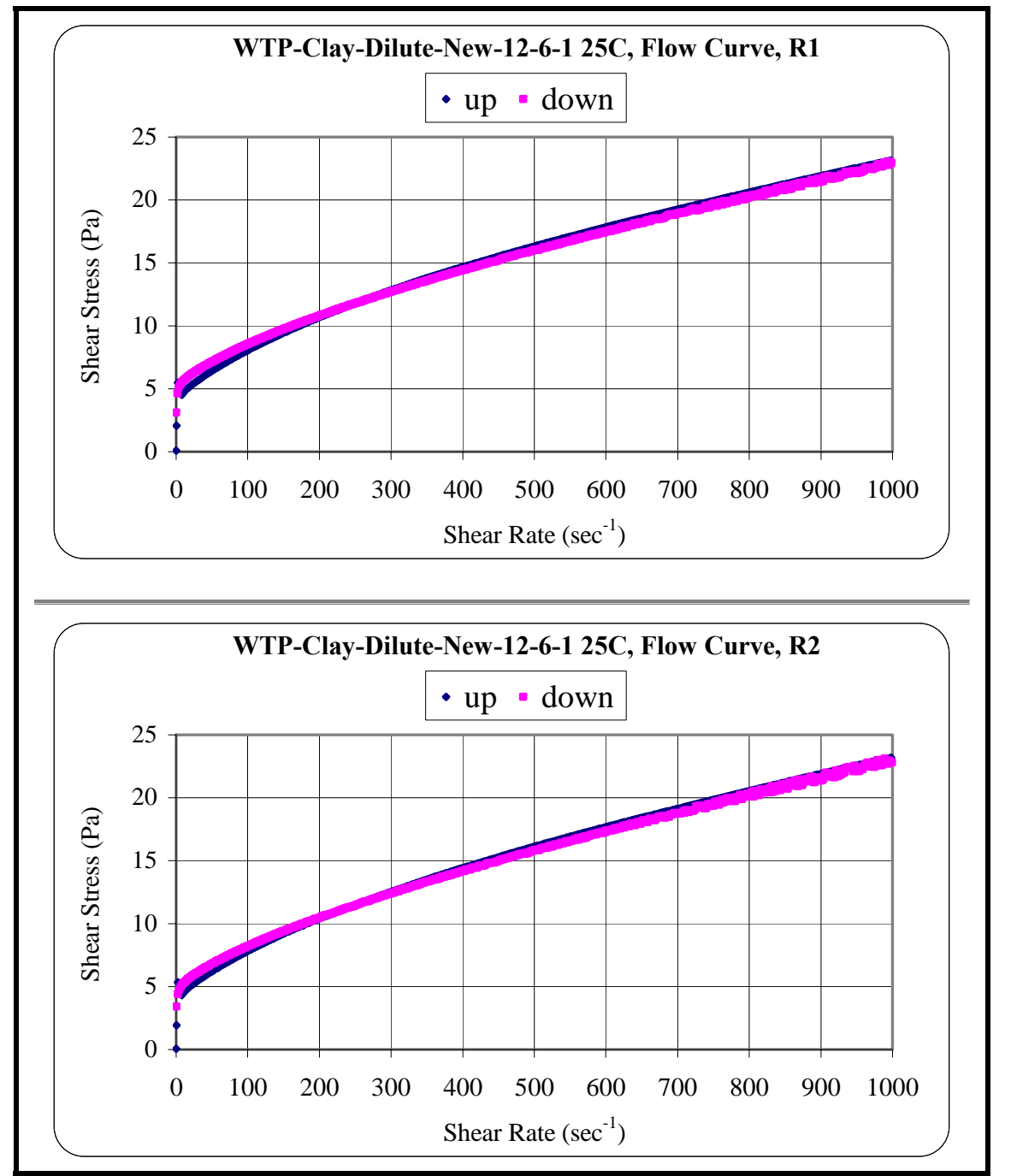

Figure A - 7: Diluted Clay to Mimic QARD Rheology New, WTPClay 2-12/6-1

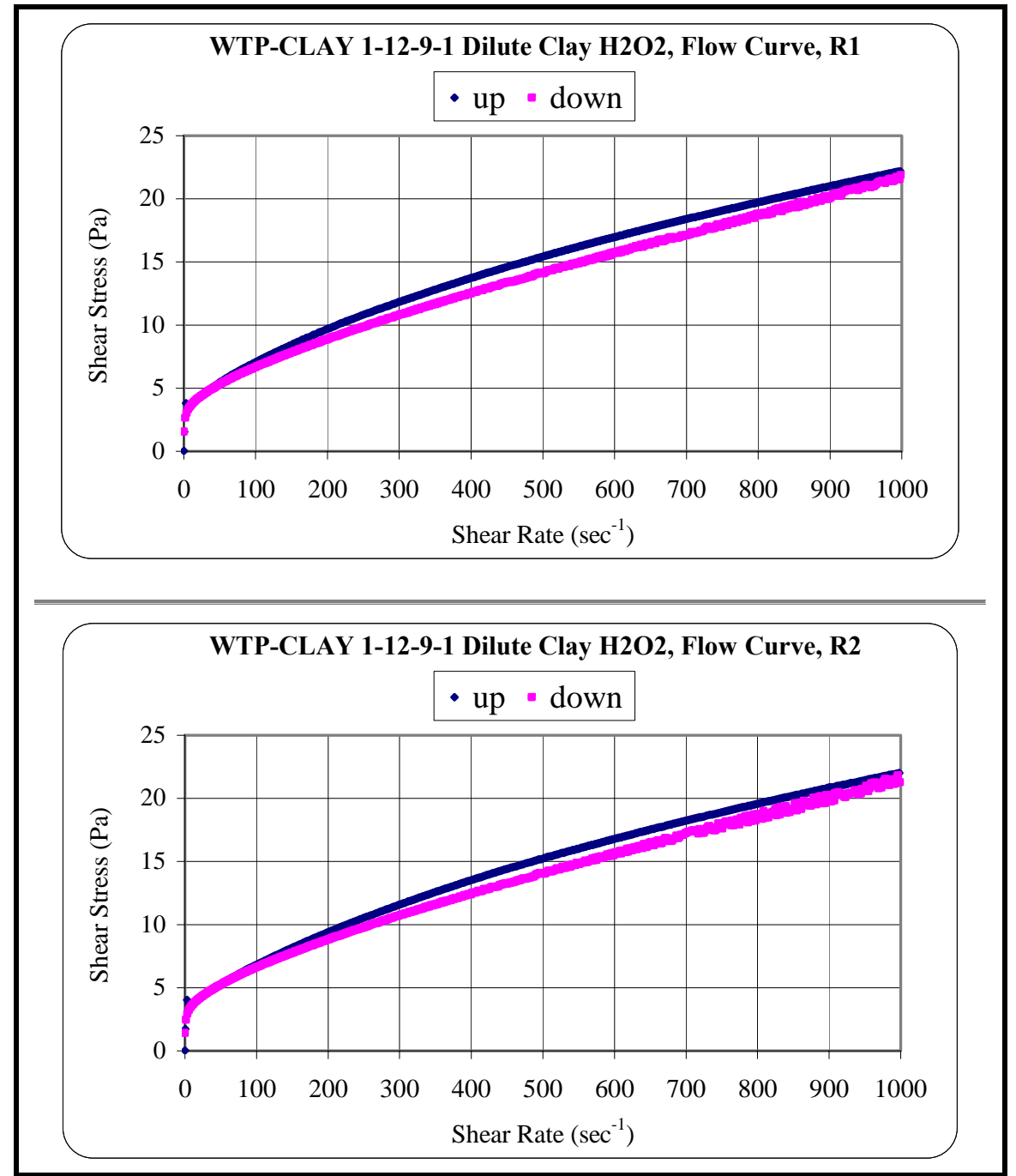

Figure A - 8: Diluted Clay After H2O2 Injection \& Release, WTP-Clay 2-12/6 
WSRC-TR-2004-00010, REV. 0 SRT-RPP-2004-00001, REV. 0

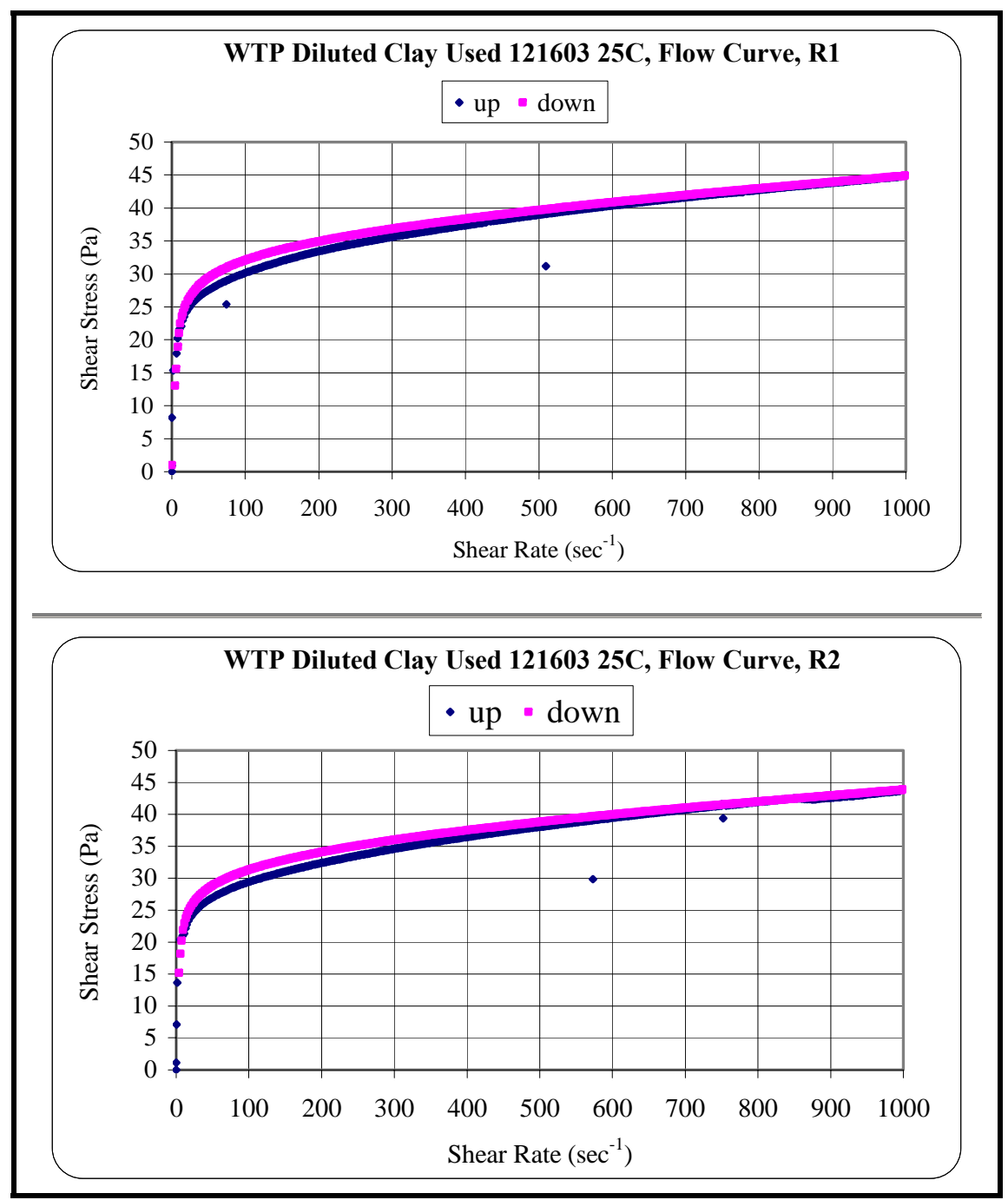

Figure A - 9: WTP-Clay-121603-Used FIU-HCET Diluted Clay
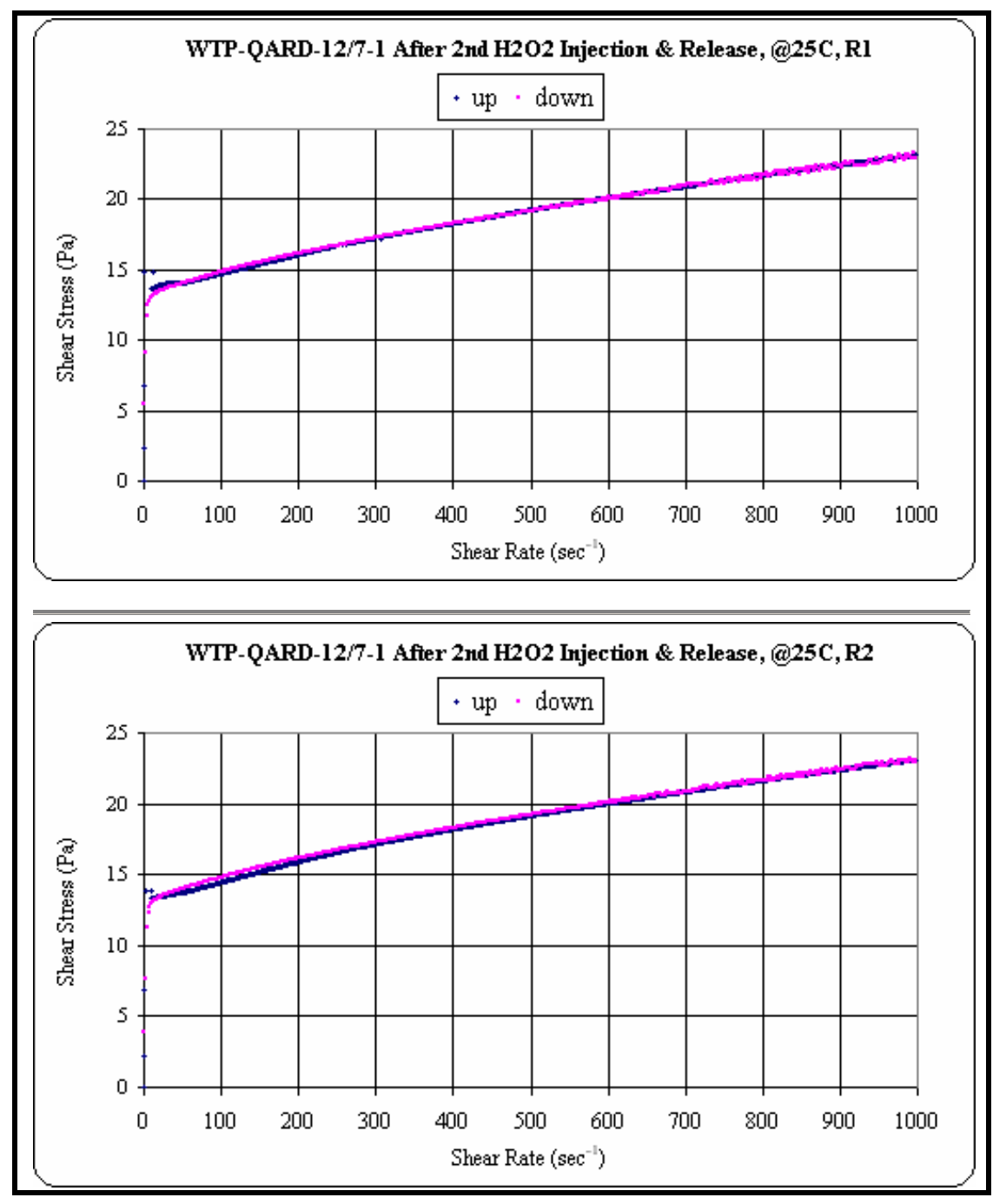

Figure A - 10: WTP-QARD-12/7-1 After $2^{\text {nd }}$ H2O2 Injection \& Release 
WSRC-TR-2004-00010, REV. 0

SRT-RPP-2004-00001, REV. 0
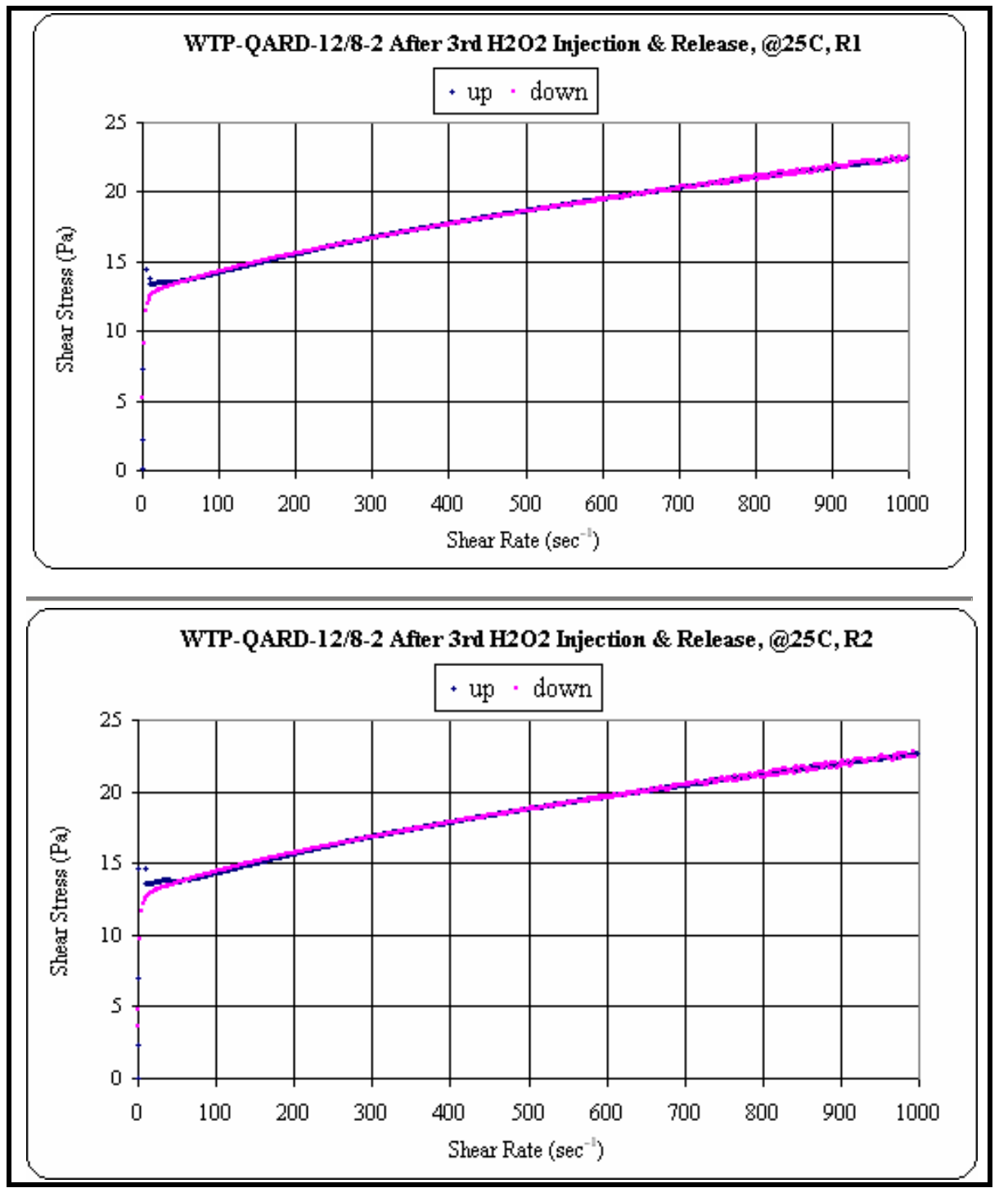

Figure A - 11: WTP-QARD-12/8-2 After $3^{\text {rd }}$ H2O2 Injection \& Release 
WSRC-TR-2004-00010, REV. 0

SRT-RPP-2004-00001, REV. 0

This page intentionally left blank. 
WSRC-TR-2004-00010, REV. 0

SRT-RPP-2004-00001, REV. 0

APPENDIX B - Vane Curves 
WSRC-TR-2004-00010, REV. 0

SRT-RPP-2004-00001, REV. 0

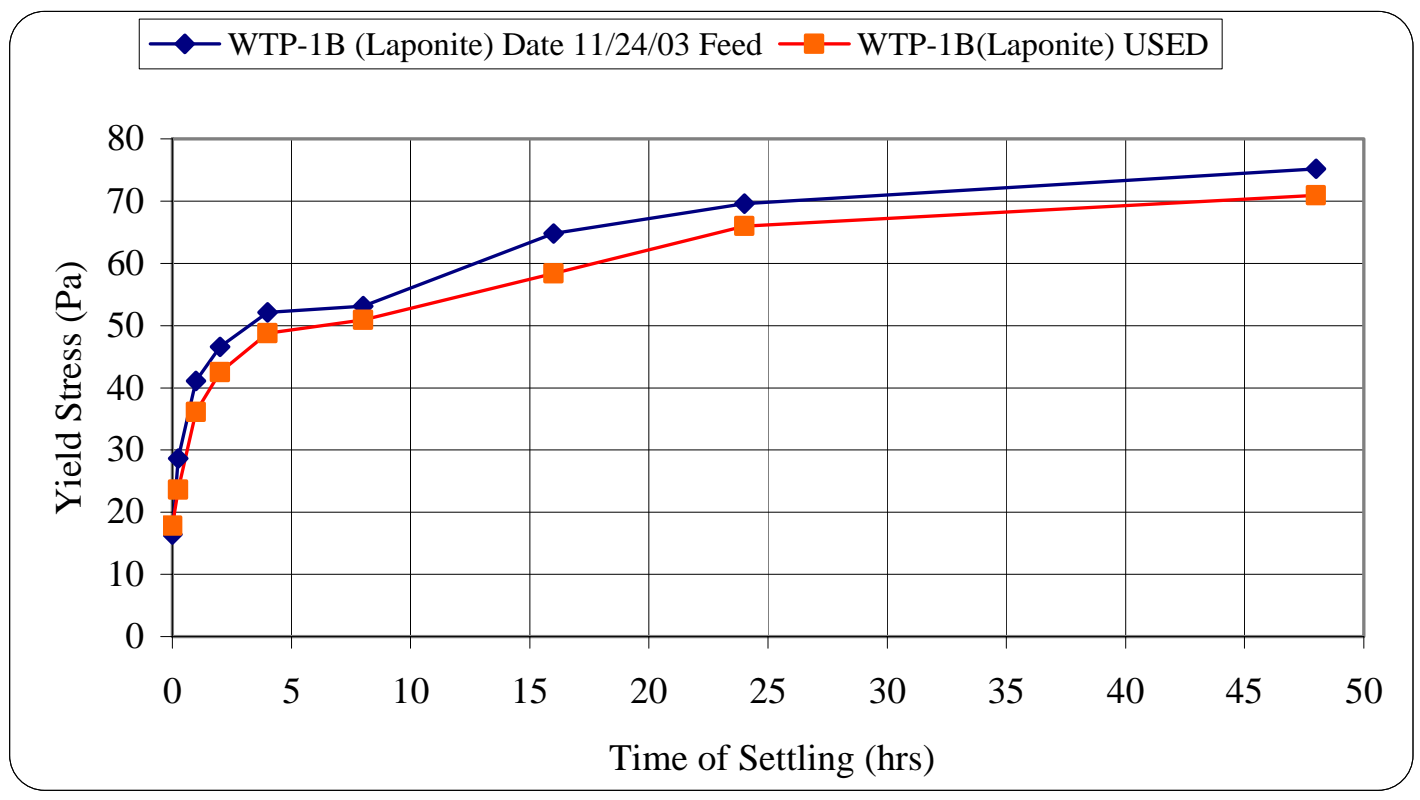

Figure B - 1: Shear Strength Results for FIU Laponite Samples

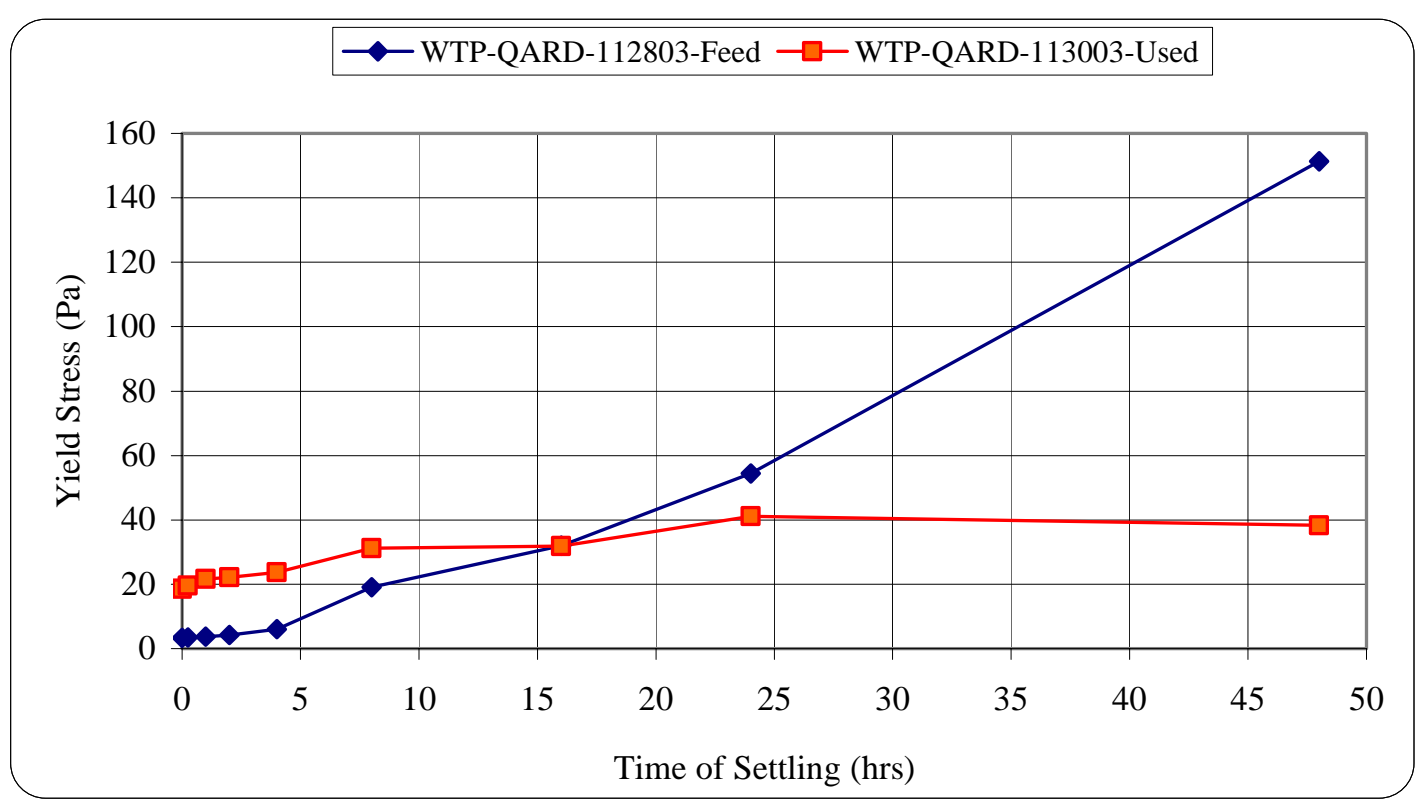

Figure B - 2: Shear Strength Results for FIU QARD Samples 
WSRC-TR-2004-00010, REV. 0

SRT-RPP-2004-00001, REV. 0

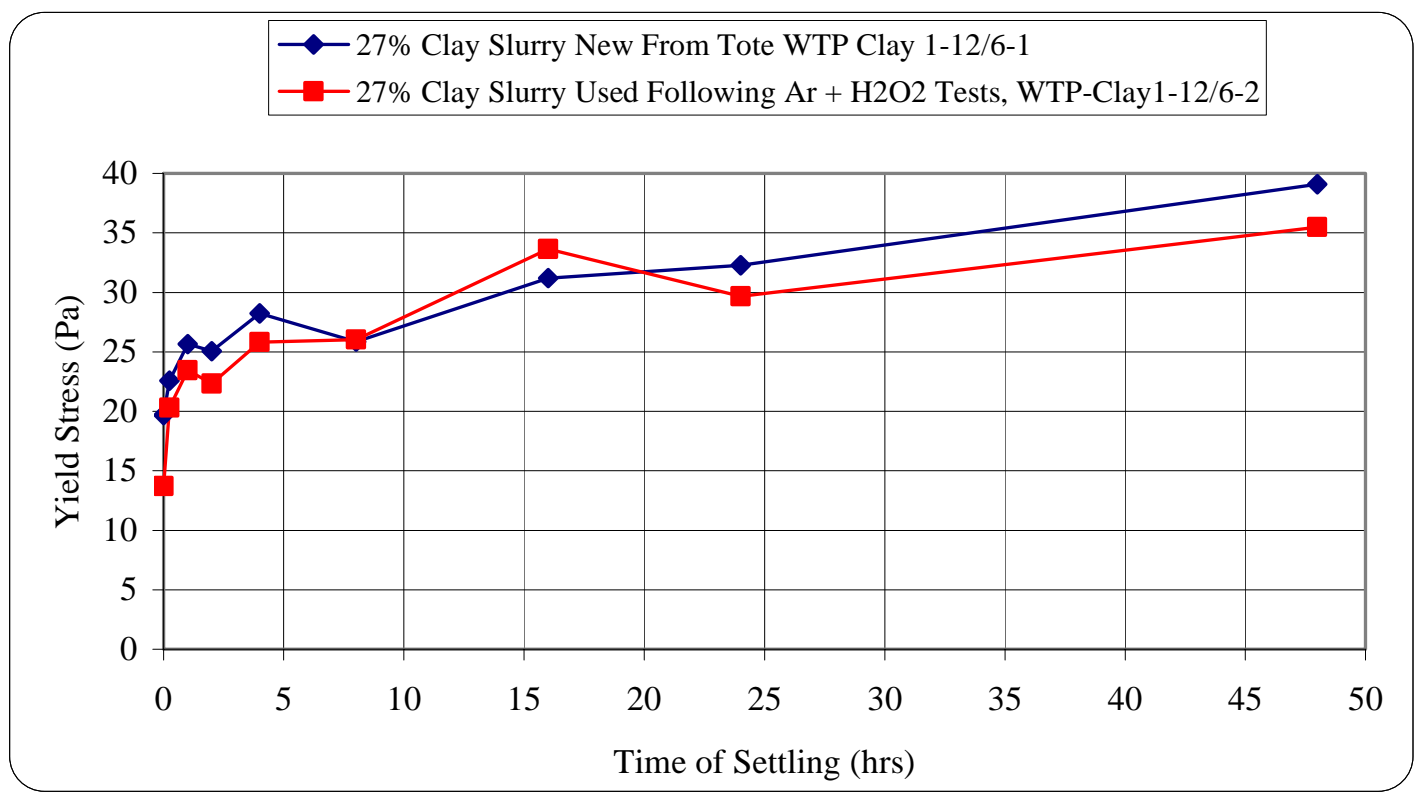

Figure B - 3: Shear Strength Results for FIU 27\% Clay Slurry

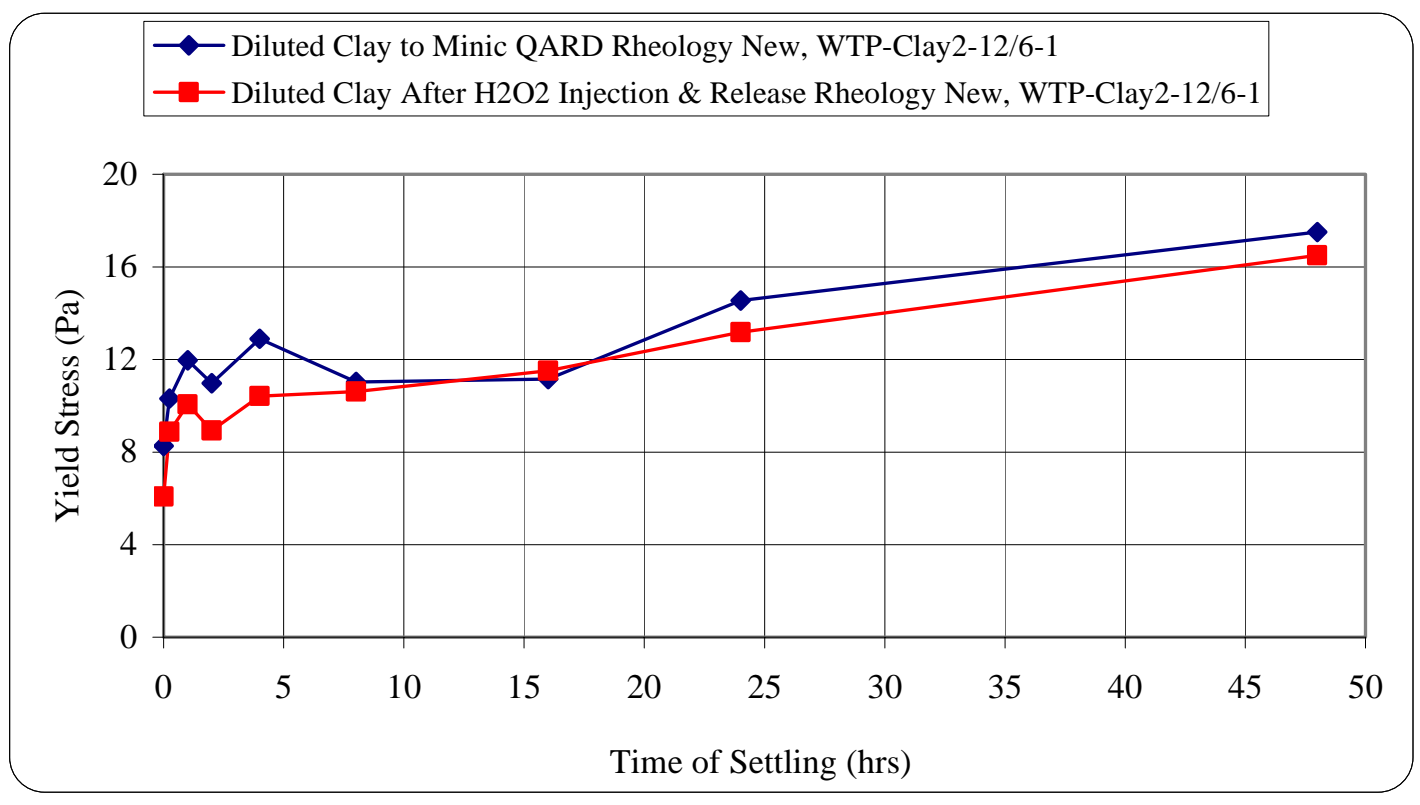

Figure B - 4: Shear Strength Results for FIU Diluted Clay to Mimic QARD Rheology 
WSRC-TR-2004-00010, REV. 0

SRT-RPP-2004-00001, REV. 0

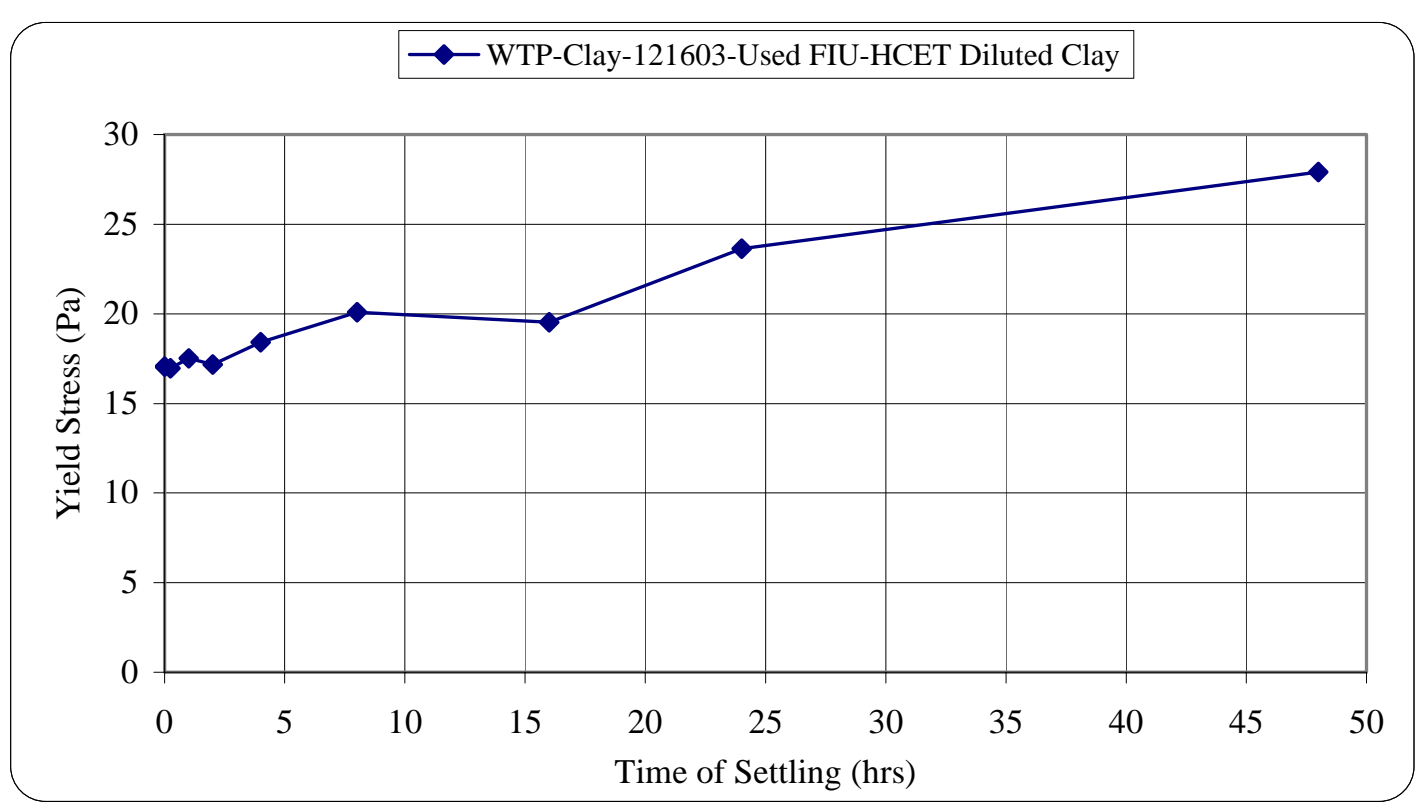

Figure B - 5: Shear Strength Results for WTP-Clay-121603-Used FIU-HCET Diluted Clay

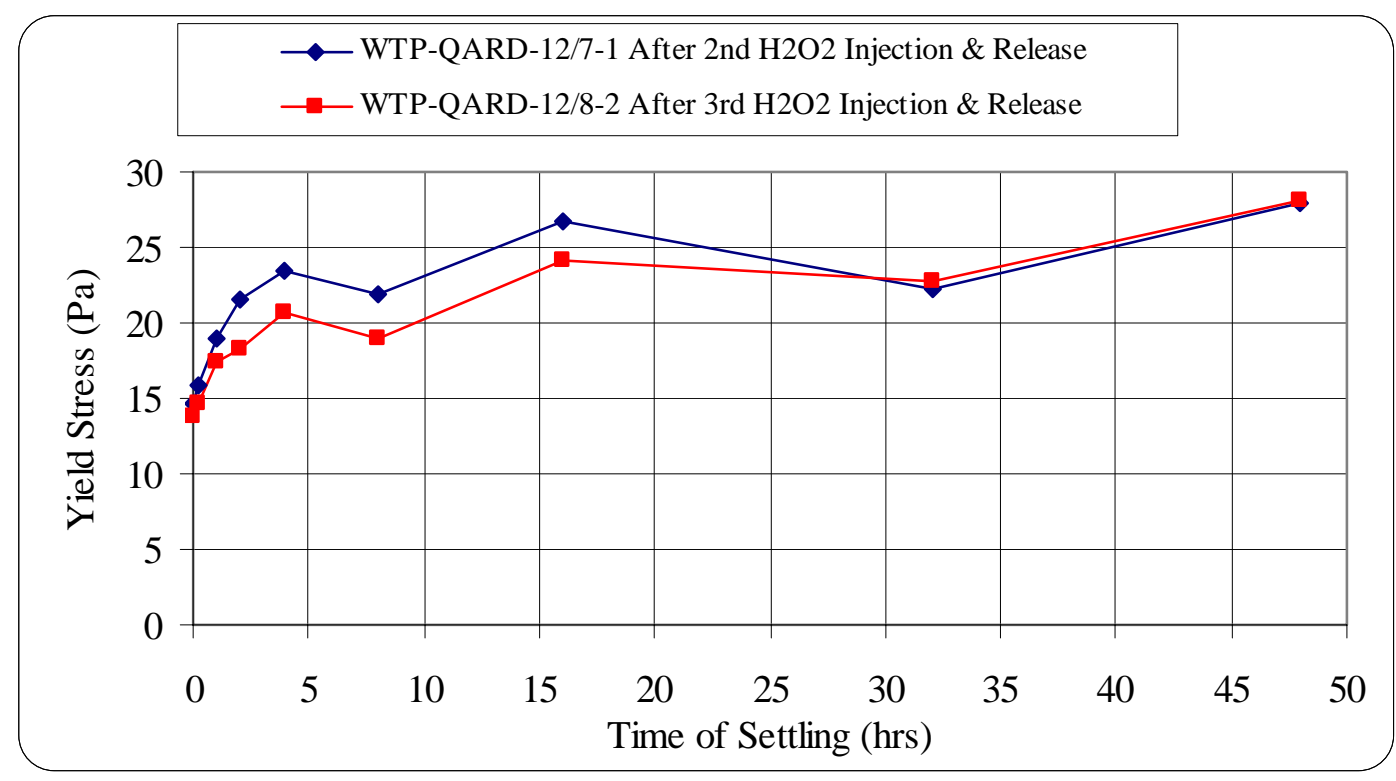

Figure B - 6: Shear Strength Results for WTP-QARD After $2^{\text {nd }}$ and $3^{\text {rd }}$ H2O2 Injection and Release Test 